US Army Corps of Engineers ${ }_{\circledast}$

Engineer Research and

Development Center

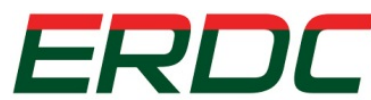

INNOVATIVE SOLUTIONS for a safer, better world

\title{
Ontology for Life-Cycle Modeling of Electrical Distribution Systems: Model View Definition
}

Kristine Fallon Associates, Inc.

June 2013

11 E. Adams Street, Suite 1100

Chicago, IL 60603

Prepared under CRADA-07-CERL-02 under the supervision of

E. William East, Project Manager (CEERD-CF-N)

Construction Engineering Research Laboratory

US Army Engineer Research and Development Center

2902 Newmark Drive

Champaign, IL 61822 
The US Army Engineer Research and Development Center (ERDC) solves the nation's toughest engineering and environmental challenges. ERDC develops innovative solutions in civil and military engineering, geospatial sciences, water resources, and environmental sciences for the Army, the Department of Defense, civilian agencies, and our nation's public good. Find out more at www.erdc.usace.army.mil.

To search for other technical reports published by ERDC, visit the ERDC online library at http://acwc.sdp.sirsi.net/client/ default. 


\title{
Ontology for Life-Cycle Modeling of Electrical Distribution Systems: Model View Definition
}

Tim Chipman

Constructivity.com LLC

2625 North Loop Drive

Ames, lowa 50010

Kristine K. Fallon, Robert A. Feldman, Gregory Williams, and Omobolawa Fadojutimi

Kristine Fallon Associates, Inc.

11 East Adams Street, Suite 1100

Chicago, IL 60603

Final report

Approved for public release; distribution is unlimited.

\author{
Prepared for US Army Corps of Engineers \\ Washington, DC 20314-1000 \\ Under Cooperative Research and Development Agreement CRADA-07-CERL-02 \\ Monitored by Construction Engineering Research Laboratory \\ US Army Engineer Research and Development Center \\ 2902 Newmark Drive \\ Champaign, IL 61822
}




\section{Abstract}

Previous efforts by the US Army Engineer Research and Development Center, Construction Engineering Research Laboratory (ERDC-CERL) to develop a life-cycle building model have resulted in the definition of a "core" building information model that contains general information describing facility assets such as spaces and equipment. To describe how facility assets (i.e., components) function together, information about assemblies of assets and their connections must also be defined. The definitions of assets, assemblies, and connections for the various buildinginformation domains are discipline-specific.

The work documented here addresses the process flow and data exchange requirements for the design of electrical distribution systems in typical Army facilities. This ontology advances the state of the art by defining an Industry Foundation Class (IFC) Model View for electrical system design supporting end users in developing compliant BIM models suggesting potential areas of automation in electrical system design.

DISCLAIMER: The contents of this report are not to be used for advertising, publication, or promotional purposes. Citation of trade names does not constitute an official endorsement or approval of the use of such commercial products. All product names and trademarks cited are the property of their respective owners. The findings of this report are not to be construed as an official Department of the Army position unless so designated by other authorized documents. 


\section{Contents}

Abstract........................................................................................................................... if

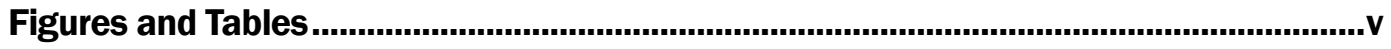

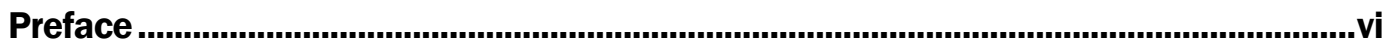

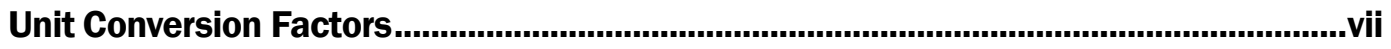

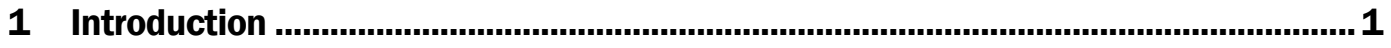

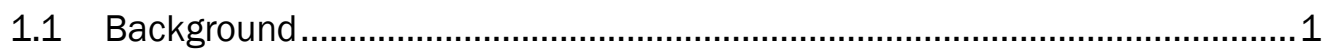

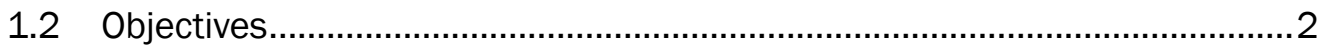

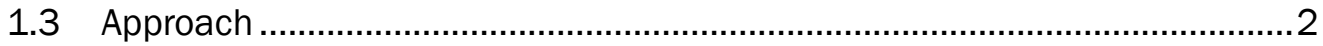

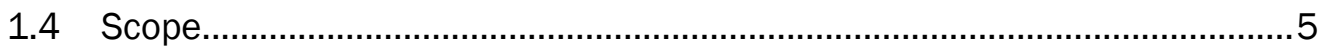

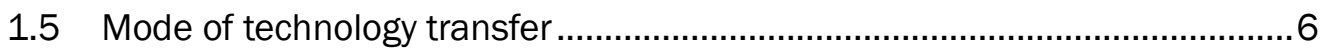

2 Electrical System Design Process Models ............................................................. 7

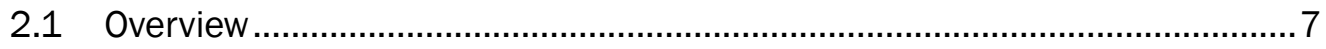

2.1.1 Electrical system design process ................................................................. 7

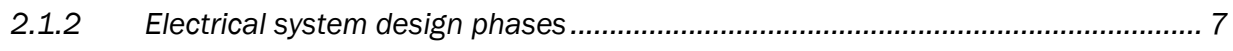

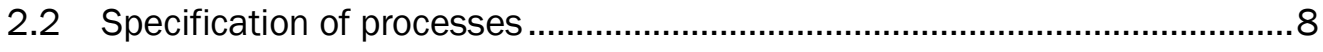

2.2.1 Criteria Phase electrical system design ............................................................... 9

2.2.2 Schematic Design Phase electrical system design............................................ 15

2.2.3 Coordinated Design Phase electrical system design .......................................... 21

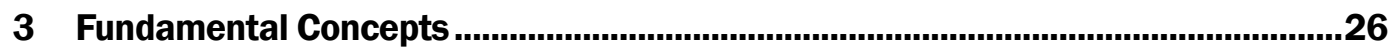

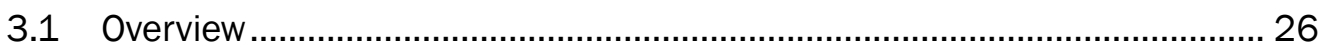

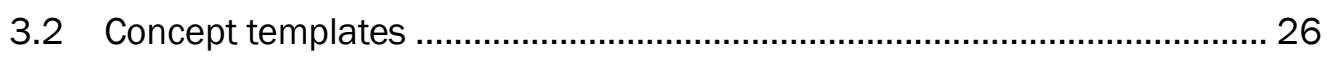

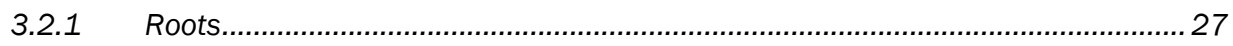

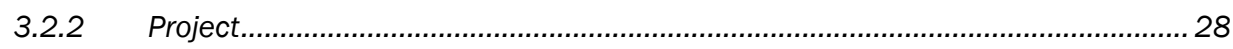

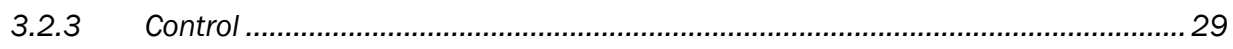

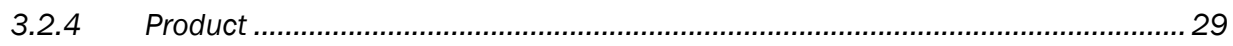

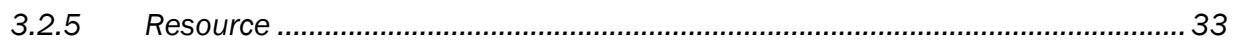

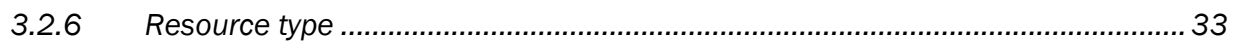

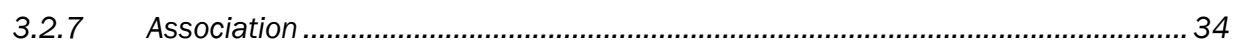

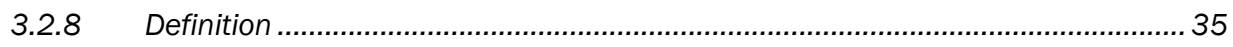

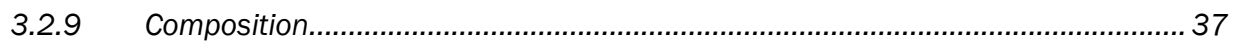

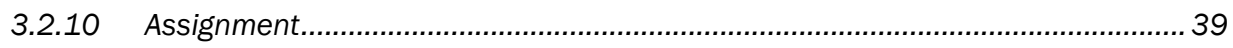

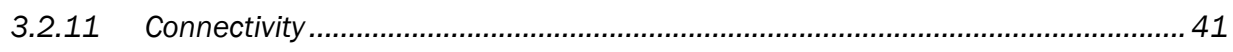

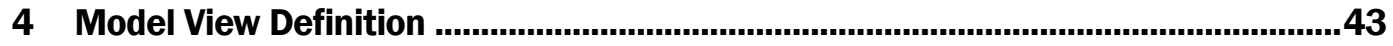

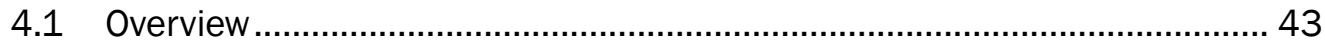

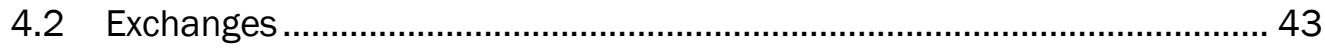

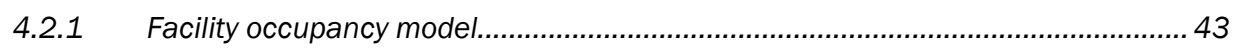




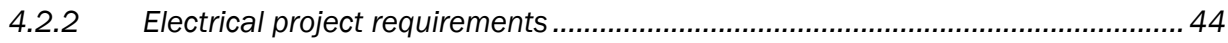

4.2.3 Equipment room requirements ...................................................................... 45

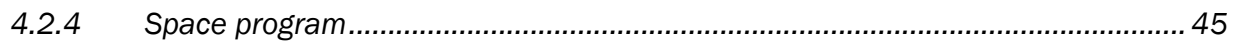

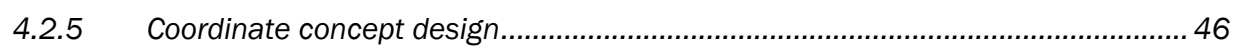

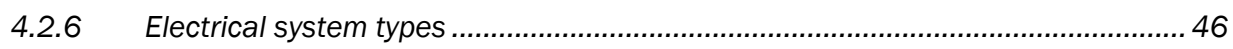

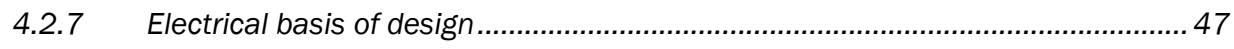

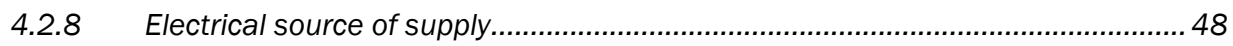

4.2.9 Electrical space requirements............................................................................... 49

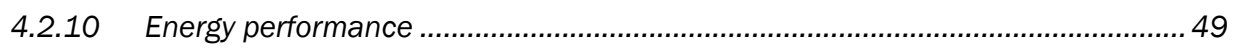

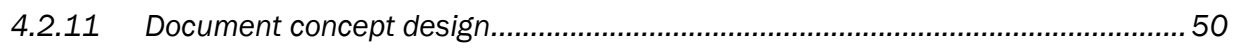

4.2.12 Locate electrical loads............................................................................... 50

4.2.13 Electrical equipment requirements............................................................. 51

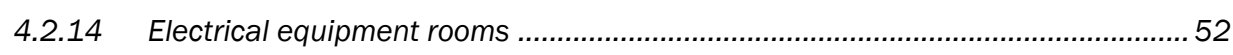

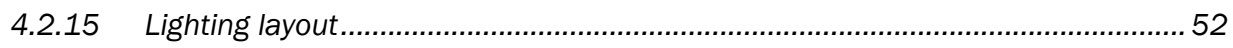

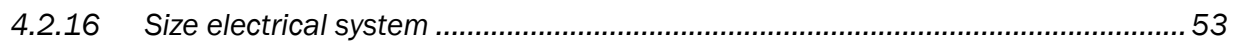

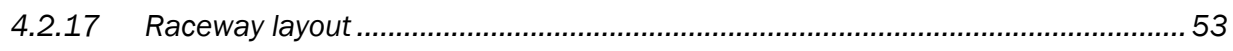

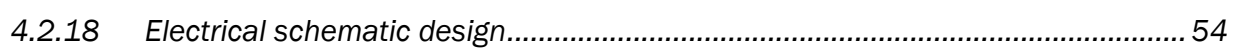

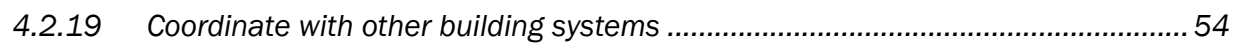

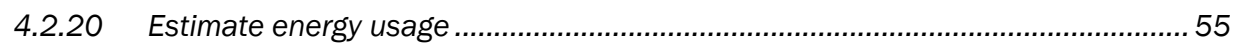

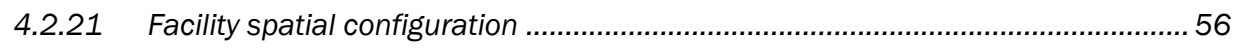

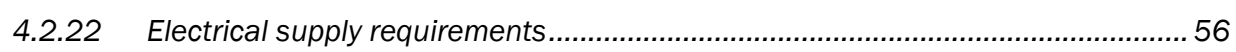

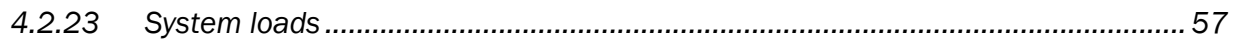

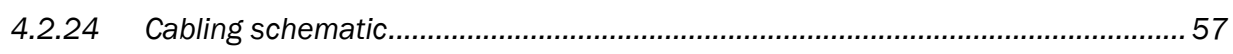

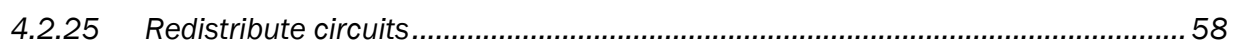

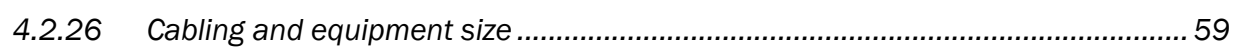

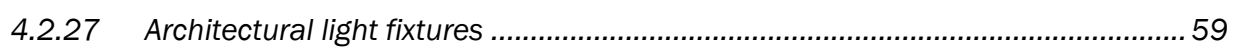

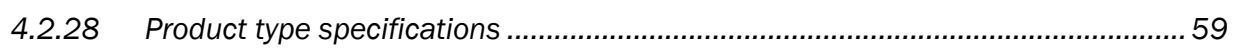

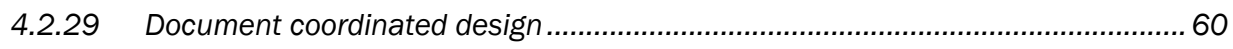

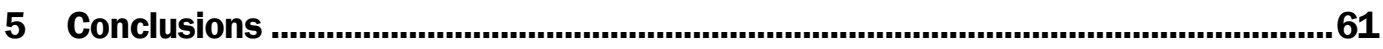

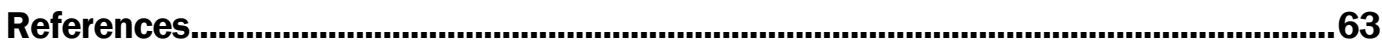

\section{Report Documentation Page}




\section{Figures and Tables}

Figures

Figure 1. Process diagram for Criteria Phase electrical system design. .............................10

Figure 2. Process diagram for Schematic Design Phase electrical system design. ............16

Figure 3. Process diagram for Coordinated Design Phase electrical system

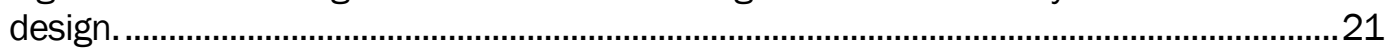

\section{Tables}

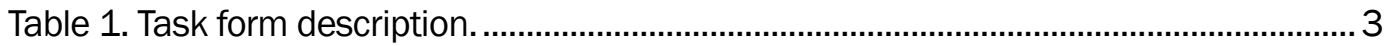

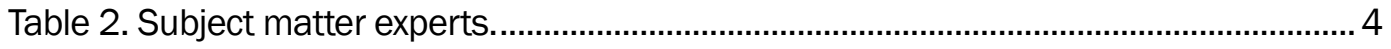




\section{Preface}

This study was conducted for the US Army Engineer Research and Development Center, Construction Engineering Research Laboratory (ERDCCERL) and the National Institute of Building Sciences (NIBS) by Kristine Fallon Associates Inc., and Constructivity.com, LLC, under CRADA-07CERL-02, "Cooperative Research and Development Agreement Between US Army Engineer Research and Development Center- Construction Engineering Laboratory and National Institute Of Building Sciences.” The CRADA supports Research, Development, Test, and Evaluation (RDT\&E) Program Element 622784 T41, "Military Facilities Engineering Technology"; Project 157249, "Life-Cycle Model For Mission Ready Sustainable Facilities (LCM)." The ERDC-CERL project manager was Dr. E. William East (CEERD-CF-N), and the NIBS project manager was Dominique Fernandez.

The work was supervised and monitored by the Engineering Processes Branch (CF-N) of the Facilities Division (CF), ERDC-CERL. Dr. East was the Project Manager. At the time of publication, Donald K. Hicks was Chief, CEERD-CF-N; L. Michael Golish was Chief, CEERD-CF; and Martin J . Savoie, CEERD-CV-ZT, was the Technical Director for Installations. The Deputy Director of ERDC-CERL was Dr. Kirankumar Topudurti and the Director was Dr. Ilker Adiguzel.

COL Kevin J . Wilson was the Commander of ERDC, and Dr. J effery P. Holland was the Director. 


\section{Unit Conversion Factors}

\begin{tabular}{|c|c|c|}
\hline Multiply & By & To Obtain \\
\hline acres & $4,046.873$ & square meters \\
\hline acre-feet & $1,233.5$ & cubic meters \\
\hline angstroms & 0.1 & nanometers \\
\hline atmosphere (standard) & 101.325 & kilopascals \\
\hline bars & 100 & kilopascals \\
\hline British thermal units (International Table) & $1,055.056$ & joules \\
\hline centipoises & 0.001 & pascal seconds \\
\hline centistokes & $1.0 \mathrm{E}-06$ & square meters per second \\
\hline cubic feet & 0.02831685 & cubic meters \\
\hline cubic inches & 1.6387064 E-05 & cubic meters \\
\hline cubic yards & 0.7645549 & cubic meters \\
\hline degrees (angle) & 0.01745329 & radians \\
\hline degrees Fahrenheit & $(\mathrm{F}-32) / 1.8$ & degrees Celsius \\
\hline fathoms & 1.8288 & meters \\
\hline feet & 0.3048 & meters \\
\hline foot-pounds force & 1.355818 & joules \\
\hline gallons (US liquid) & $3.785412 \mathrm{E}-03$ & cubic meters \\
\hline hectares & $1.0 \mathrm{E}+04$ & square meters \\
\hline horsepower (550 foot-pounds force per second) & 745.6999 & watts \\
\hline inches & 0.0254 & meters \\
\hline inch-pounds (force) & 0.1129848 & newton meters \\
\hline kilotons (nuclear equivalent of TNT) & 4.184 & terajoules \\
\hline knots & 0.5144444 & meters per second \\
\hline microinches & 0.0254 & micrometers \\
\hline microns & $1.0 \mathrm{E}-06$ & meters \\
\hline miles (nautical) & 1,852 & meters \\
\hline miles (US statute) & $1,609.347$ & meters \\
\hline miles per hour & 0.44704 & meters per second \\
\hline mils & 0.0254 & millimeters \\
\hline ounces (mass) & 0.02834952 & kilograms \\
\hline ounces (US fluid) & $2.957353 \mathrm{E}-05$ & cubic meters \\
\hline pints (US liquid) & 4.73176 E-04 & cubic meters \\
\hline
\end{tabular}




\begin{tabular}{|c|c|c|}
\hline Multiply & By & To Obtain \\
\hline pints (US liquid) & 0.473176 & liters \\
\hline pounds (force) & 4.448222 & newtons \\
\hline pounds (force) per foot & 14.59390 & newtons per meter \\
\hline pounds (force) per inch & 175.1268 & newtons per meter \\
\hline pounds (force) per square foot & 47.88026 & pascals \\
\hline pounds (force) per square inch & 6.894757 & kilopascals \\
\hline pounds (mass) & 0.45359237 & kilograms \\
\hline pounds (mass) per cubic foot & 16.01846 & kilograms per cubic meter \\
\hline pounds (mass) per cubic inch & $2.757990 \mathrm{E}+04$ & kilograms per cubic meter \\
\hline pounds (mass) per square foot & 4.882428 & kilograms per square meter \\
\hline pounds (mass) per square yard & 0.542492 & kilograms per square meter \\
\hline quarts (US liquid) & 9.463529 E-04 & cubic meters \\
\hline slugs & 14.59390 & kilograms \\
\hline square feet & 0.09290304 & square meters \\
\hline square inches & $6.4516 \mathrm{E}-04$ & square meters \\
\hline square miles & $2.589998 E+06$ & square meters \\
\hline square yards & 0.8361274 & square meters \\
\hline tons (force) & $8,896.443$ & newtons \\
\hline tons (force) per square foot & 95.76052 & kilopascals \\
\hline tons (long) per cubic yard & $1,328.939$ & kilograms per cubic meter \\
\hline tons (nuclear equivalent of TNT) & 4.184E+09 & joules \\
\hline tons ( 2,000 pounds, mass) & 907.1847 & kilograms \\
\hline tons ( 2,000 pounds, mass) per square foot & $9,764.856$ & kilograms per square meter \\
\hline yards & 0.9144 & meters \\
\hline
\end{tabular}




\section{Introduction}

\subsection{Background}

Previous efforts by the US Army Engineer Research and Development Center, Construction Engineering Research Laboratory (ERDC-CERL) to develop a life-cycle building model have resulted in the definition of a "core" building information model that contains general information describing facility assets such as spaces and equipment (East, Love, and Nisbet 2010). To describe how facility assets (i.e., components) function together, information about assemblies of assets and their connections must also be defined. The definitions of assets, assemblies, and connections for the various building-information domains are discipline-specific. Taken together, studies of all essential building-information domains will create a unified framework for developing automatic design checks, ensuring construction compliance, improving operations and maintenance efficiency, and evaluating alternatives for redesign within completed facilities.

COBie (East 2012a) was the first step in analyzing information exchanges in the life cycle of a building. Since March 2012, COBie has been part of the National BIM Standard- United States (NBIMS-US). COBie defines the format for providing information about building assets from the planning phase through design, construction, and operations. Properties of these assets may also be captured in the COBie date exchange format. The COBie Guide, a commentary on the COBie standard (public draft downloadable at link from http://www.nibs.org/?page=bsa_cobieguide), does not prescribe how to model specific assemblies of components or how components and assemblies are connected (East 2007, East 2012a). Those aspects of modeling and information exchange require a domain-specific ontology for every system needed to construct a functional building.

The work documented here addresses the process flow and data exchange requirements for the design of electrical distribution systems in typical Army facilities. This ontology advances the state of the art by

1. defining an Industry Foundation Class (IFC) Model View for electrical system design

2. supporting end users in developing compliant BIM models

3. suggesting potential areas of automation in electrical system design. 


\subsection{Objectives}

The objectives of the present work were to identify and document the requirements for building electrical system design for the purpose of creating formal specifications that can be directly applied to open-standards building information models (BIM) at the coordinated design stage of building construction.

\subsection{Approach}

To document the process and exchange requirements, the team followed the Information Delivery Manual (IDM) and Model View Definition (MVD) procedures defined by the International Organization for Standardization (ISO) and buildingSmart International (e.g., Wix 2007, Hietanen 2008). Validation of the process diagrams and exchange requirements followed the process outlined below:

1. Create drafts of process diagrams and task descriptions for each of three phases of the design process - Criteria (i.e., Programming and Concept Design), Schematic Design (i.e., Design Development), and Coordinated Design (i.e., Construction Documents). The draft process diagrams included suggested steps for the typical Army design process, and the task descriptions included suggested information requirements needed to accomplish the task step.

2. Assemble a group of subject matter experts (SMEs) to review and comment on the draft process diagrams and task descriptions. This group included two architects, two engineers, and two specifiers with experience in the design of building electrical systems.

3. Meet with the SME reviewers to explain the process and review criteria.

4. Send the process diagrams and task descriptions to the SMEs for their review.

5. Analyze the SME comments and contact the SMEs for clarification and additional comments, as needed.

6. Revise the process diagrams and task descriptions based on the SME comments.

The research team also consulted a publication on ELie (East 2012c) and unpublished research notes on the exploratory modeling of electrical system components and connections, called SPARKie (East 2012b). 
The specific selection and sequencing of tasks was intended as a starting point that would be refined using the SME reviewers' feedback. The task forms included the information summarized in Table 1

Table 1. Task form description.

\begin{tabular}{|l|l|}
\hline \multicolumn{1}{|c|}{ Item } & \multicolumn{1}{c|}{ Description } \\
\hline Task ID & Sequential ID number for the task. \\
\hline Task Name & A short descriptive name for the task \\
\hline $\begin{array}{l}\text { Information Provider } \\
\text { (Roles Involved) }\end{array}$ & $\begin{array}{l}\text { The role or roles that provide the input information } \\
\text { necessary to do the task. }\end{array}$ \\
\hline $\begin{array}{l}\text { Information Provider } \\
\text { (Phase) }\end{array}$ & $\begin{array}{l}\text { The stage in the process when the required information } \\
\text { is created. }\end{array}$ \\
\hline Actor (Roles Involved) & The role or roles that complete the task. \\
\hline Actor (Phase) & $\begin{array}{l}\text { The stage in the process at which the task requires the } \\
\text { information. }\end{array}$ \\
\hline Information Required & The input information necessary to complete the task. \\
\hline Current Methods & $\begin{array}{l}\text { A short description of the task and its inputs and } \\
\text { outputs. }\end{array}$ \\
\hline
\end{tabular}

The experts were asked to review the tasks with the following questions in mind:

- Do the task forms accurately and completely detail all information needed to perform the task?

0 If not, what is missing?

o Who provides the additional inputs?

- Are current methods of performing the task accurately described?

For the process diagrams, the reviewers were asked:

- Although every project has unique circumstances, are the tasks shown in the typically correct order?

- Have we missed any tasks?

- Are there any unnecessary tasks?

- Are all tasks assigned to the correct phase(s)?

- Are all tasks assigned to the correct actor?

- Are all actors that provide the Information Required indicated?

- Are any extraneous actors indicated?

Table 2 lists the expert reviewers and pertinent background information. 
Table 2. Subject matter experts.

\begin{tabular}{|c|c|c|}
\hline Name & Organization & Area of Expertise \\
\hline \multirow{2}{*}{$\begin{array}{l}\text { Randy Deutsch AIA, LEED } \\
\text { AP }\end{array}$} & Deutsch Insights, Inc. & Architect \\
\hline & \multicolumn{2}{|c|}{$\begin{array}{l}\text { Senior Architectural Designer \& Associate Principal with } \\
\text { proven track record for design leadership. Demonstrated } \\
\text { success designing and managing complex projects } \\
\text { including high-rises, retail, mixed-use developments, } \\
\text { housing \& master plans. Professional thought leader, } \\
\text { presenter, instructor, mentor \& author. Instrumental in } \\
\text { firm-wide BIM and IPD adoption \& implementation. AIA } \\
\text { Young Architect Award recipient. University building } \\
\text { technology, design studio \& professional practice } \\
\text { instructor. Author of BIM and Integrated Design (Wiley } \\
\text { 2011). }\end{array}$} \\
\hline \multirow{2}{*}{$\begin{array}{l}\text { Susan F. King, FAIA, LEED } \\
\text { AP BD+C }\end{array}$} & Harley Ellis Devereaux & Architect \\
\hline & \multicolumn{2}{|c|}{$\begin{array}{l}\text { As a principal with Harley Ellis Devereaux, Susan King is } \\
\text { the firm's National Sustainable Design Leader, } \\
\text { developing and implementing nationwide design policies } \\
\text { in regards to sustainability. A practicing architect with } 25 \\
\text { years of experience, she has lead numerous multi- } \\
\text { disciplinary sustainable developments teams on projects } \\
\text { ranging in scale from urban infill for newly constructed } \\
\text { housing to the repurposing of existing structures to the } \\
\text { master planning study of the } 35 \text { acre Chicago } 2016 \\
\text { Olympic Village site. A true collaborator, Susan joins } \\
\text { design teams with ease and a practical approach to the } \\
\text { integrated design process. An advocate for green market } \\
\text { transformation of the building industry, Ms. King is } \\
\text { routinely invited to speak on sustainable design topics. }\end{array}$} \\
\hline \multirow[t]{2}{*}{ Jim Forester } & Newforma, Inc. & Engineer \\
\hline & \multicolumn{2}{|c|}{$\begin{array}{l}\text { California P.E. license M24307. Co-Founder and Senior } \\
\text { Technical Advisor at Newforma, Inc. Original member of } \\
\text { the buildingSmart International Model Support Group. I } \\
\text { was involved with the many of the original definitions of } \\
\text { the building services concepts and how they are } \\
\text { connected, including the underlying graph } \\
\text { representations supporting both symbolic and physical } \\
\text { connectivity that would support mass and energy flow } \\
\text { simulations. }\end{array}$} \\
\hline
\end{tabular}




\begin{tabular}{|c|c|c|}
\hline Name & Organization & Area of Expertise \\
\hline \multirow[t]{2}{*}{ Kenneth Solvik } & Data Design System & Engineer \\
\hline & \multicolumn{2}{|c|}{$\begin{array}{l}\text { Master of Management. Electrical Engineer, specialized } \\
\text { field installation, automation, building control and } \\
\text { programming. } \\
\text { CTO and R\&D coordinator. Manager and participant in } \\
\text { multiple R\&D projects. IFC (EL-1 \& EL-2), MEP Quantity } \\
\text { Take Off (IDM), MEP Fast and Easy planning, Innovative } \\
\text { MEP design and simulations process, BIM enhancer \& } \\
\text { BIM energy efficiency optimizer. }\end{array}$} \\
\hline \multirow{2}{*}{$\begin{array}{l}\text { Mark Kalin FAIA FCSI } \\
\text { LEED }\end{array}$} & Kalin Associates & Specifier \\
\hline & \multicolumn{2}{|c|}{$\begin{array}{l}\text { Registered architect, CSI-certified construction specifier, } \\
\text { LEED-accredited professional, and one of only } 27 \\
\text { individuals ever advanced to fellowship in both the } \\
\text { American Institute of Architects and the Construction } \\
\text { Specifications Institute. Author of numerous publications } \\
\text { on specifications, product selection, and green specs, } \\
\text { who has presented more than } 100 \text { sessions at regional } \\
\text { and national conventions. He has taught architectural } \\
\text { specifications at Harvard University Graduate School of } \\
\text { Design and is currently chair of the Sustainable Facilities } \\
\text { Practice Group of the Construction Specifications } \\
\text { Institute. }\end{array}$} \\
\hline \multirow[t]{2}{*}{ Chris Nelson } & Nelson Electric & Specifier \\
\hline & \multicolumn{2}{|c|}{$\begin{array}{l}\text { Chris specifies and designs electrical systems on } \\
\text { commercial and industrial building projects at Nelson } \\
\text { Electric in Ames, lowa. Previously, he served as } \\
\text { Enterprise Systems Manager at The Weitz Company for } 9 \\
\text { years, where he had significant experience leading } \\
\text { Building Information Modeling initiatives. He was also a } \\
\text { Project Engineer at The Meyne Company for } 2 \text { years. He } \\
\text { has an MBA from the University of lowa, an MS in Civil \& } \\
\text { Construction Engineering from lowa State University, and } \\
\text { a BS in Chemical Engineering from lowa State University. }\end{array}$} \\
\hline
\end{tabular}

\subsection{Scope}

The scope of the work documented here was to diagram the electrical system design process, and to identify and document the relevant data exchanges. A separate report (ERDC/CERL CR-13-3) applies this ontology to the updating of three previously developed experimental BIM models using commercial off-the-shelf (COTS) software. Those models represent three types of typical low-rise Army facilities: a duplex apartment, an office building, and a medical clinic. The experimental application work 
identifies some current product limitations in achieving successful information exchange.

\subsection{Mode of technology transfer}

Documentation of this ontology will be used as the basis for a ballot submission to the National BIM Standard- United States. Model files created for the related validation application (ERDC/CERL CR-13-3) will be made publicly available for testing and evaluation of the proposed open BIM standard that results from this work. 


\section{Electrical System Design Process Models}

\subsection{Overview}

Building design is a highly iterative process during which information is gathered, design options are evaluated and selections are made. The goal is to achieve a final design in which aesthetics, cost and systems performance are all optimized. During design, each choice has multiple effects. Optimized design can only be achieved through multiple iterations of interdependent analyses.

Today's designers and owners seek to optimize multiple aspects of a building, including first cost, life cycle cost and environmental impact. Early adopters of building information modeling technology have demonstrated that the use of computable building models, coupled with the availability of analysis software, facilitates and reduces cycle times of the iterations necessary to achieve such optimization (Fallon and Palmer 2007). The purpose of this electrical systems ontology is to define a standardized computable description of all electrical system parameters necessary for a complete design. The availability of such a standardized, computable description supports the development and use of electrical system design automation software.

\subsubsection{Electrical system design process}

The design of building electrical systems iterates through multiple steps, involving multiple parties and the repeated refinement of the design as it moves from generalized concepts and equipment types to detailed construction documents with the required equipment specified. The process diagrams in this document focus on the design tasks and data exchanges involving the Architect and Electrical Engineer. Data required from other project participants are also documented.

\subsubsection{Electrical system design phases}

The design process documented in this report is divided into three general phases, typical of the Design-Bid-Build process for USACE projects. Although the sequence of tasks and even the actors for each task can vary, depending on project delivery approach and on the internal organization 
of the professional services provider company(ies), the tasks that must be completed and the information required remain constant.

\subsubsection{Criteria (Programming and Concept Design)}

The Criteria phase requires gathering the necessary information that will define the project's scope, budget, and overall goals. The Owner's Project Requirements (OPR), building codes, site location, and sustainability goals are all indentified during this phase. Once the building program has been developed, the Facility Occupancy Model can be determined. This information allows the Architect and Electrical Engineer to develop a Concept Design. Typically, several options are created to compare designs or system alternatives.

\subsubsection{Schematic design (Design Development)}

The Schematic Design phase requires using the information developed during the Criteria phase to develop the building design further. For electrical systems design, most of the information is generated by the Electrical Engineer. The Architect provides information regarding electrical load types and locations. Other consultants will provide electrical requirements for other building systems. This information allows the Electrical Engineer to determine the overall electricity demand. During this phase, specifications for the anticipated equipment are developed in addition to the drawings. The specifications identify performance requirements for the various electrical system components.

\subsubsection{Coordinated design (Construction Documents)}

The Coordinated Design phase involves finalizing the documents in preparation for bidding and construction. Primarily, this involves updating the drawings and specifications completed in the previous phases with more detailed, accurate information about the building and systems. Again, this requires that the Electrical Engineer receive input from the Architect and any others involved whose particular discipline could have an impact on the electrical system design.

\subsection{Specification of processes}

This section contains three Process Diagrams covering the electrical system design phases of (1) Criteria (Programming and Concept Design), (2) Schematic Design (Design Development) and (3) Coordinated Design 
(Construction Documents). These phases have been assigned an arbitrary sequential number $(10,20$ or 30$)$ to aid in tracking and coordinating tasks. Following each of the three diagrams are tabular descriptions of the tasks shown in each diagram.

The diagrams and task descriptions have been revised to reflect the reviews and comments made by the SMEs. Several of the reviewers suggested alternative process flows, based on their experience with specific types of projects and project delivery approaches - Design-Build versus DesignBid-Build, for example. The suggestions were evaluated and, in some cases, the original flow was modified. Even where the workflow differed, however, the design tasks and information requirements have remained the same.

The solidification of the design involves an iterative process, where the owner, architect, the plumbing engineer and other specialists must reconcile their needs with those of others. An explicit understanding of the process and its information requirements can help streamline the process by focusing on what exchanges take place and who is affected. It can also be used to help define new ways of reviewing multiple design options and integrating them into the overall process.

The detailed Exchange Requirements derived from the following task descriptions are described in the next chapter.

\subsubsection{Criteria Phase electrical system design}

The Criteria Phase consists of the following tasks, shown diagrammatically in Figure 1. 
Figure 1. Process diagram for Criteria Phase electrical system design.

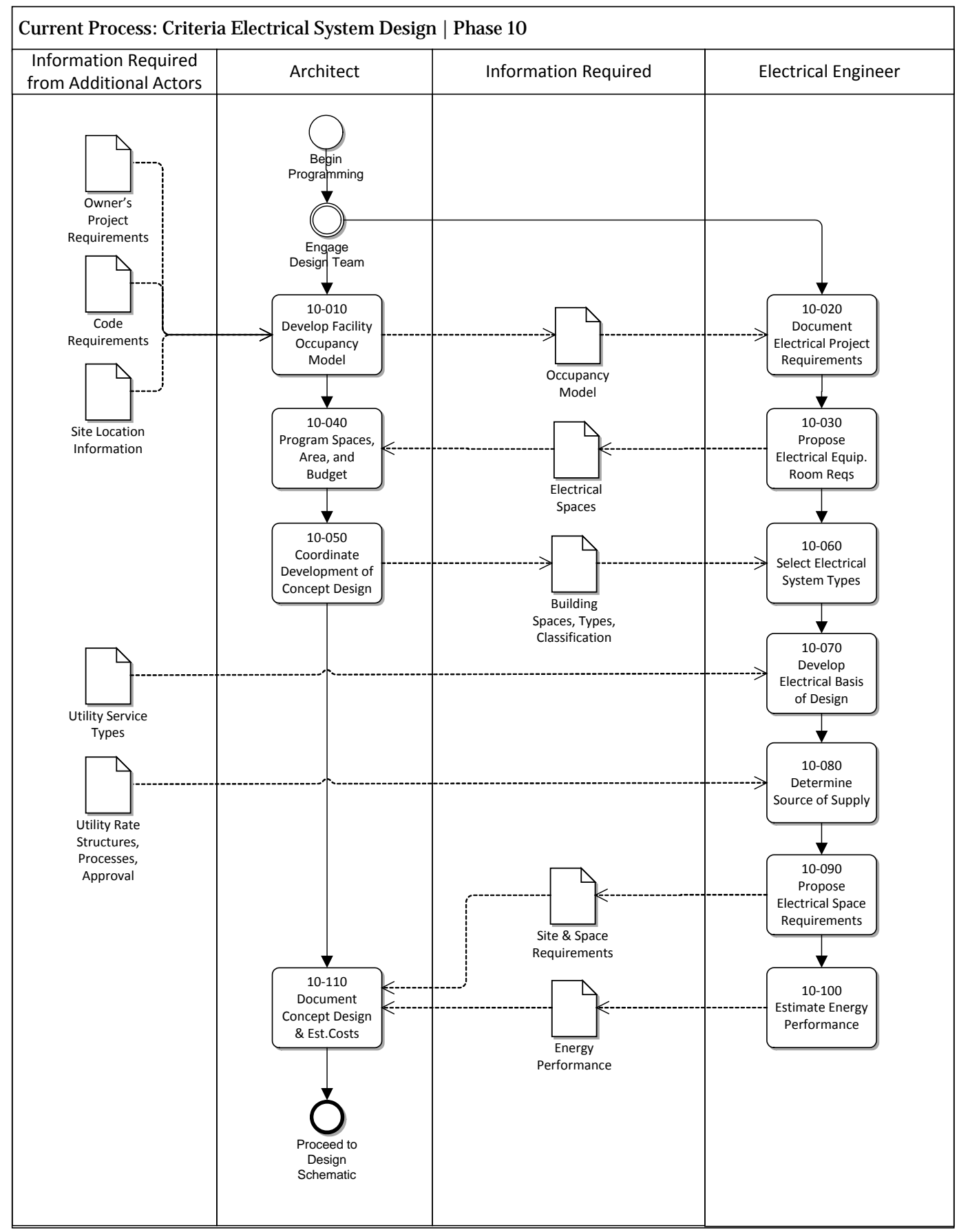




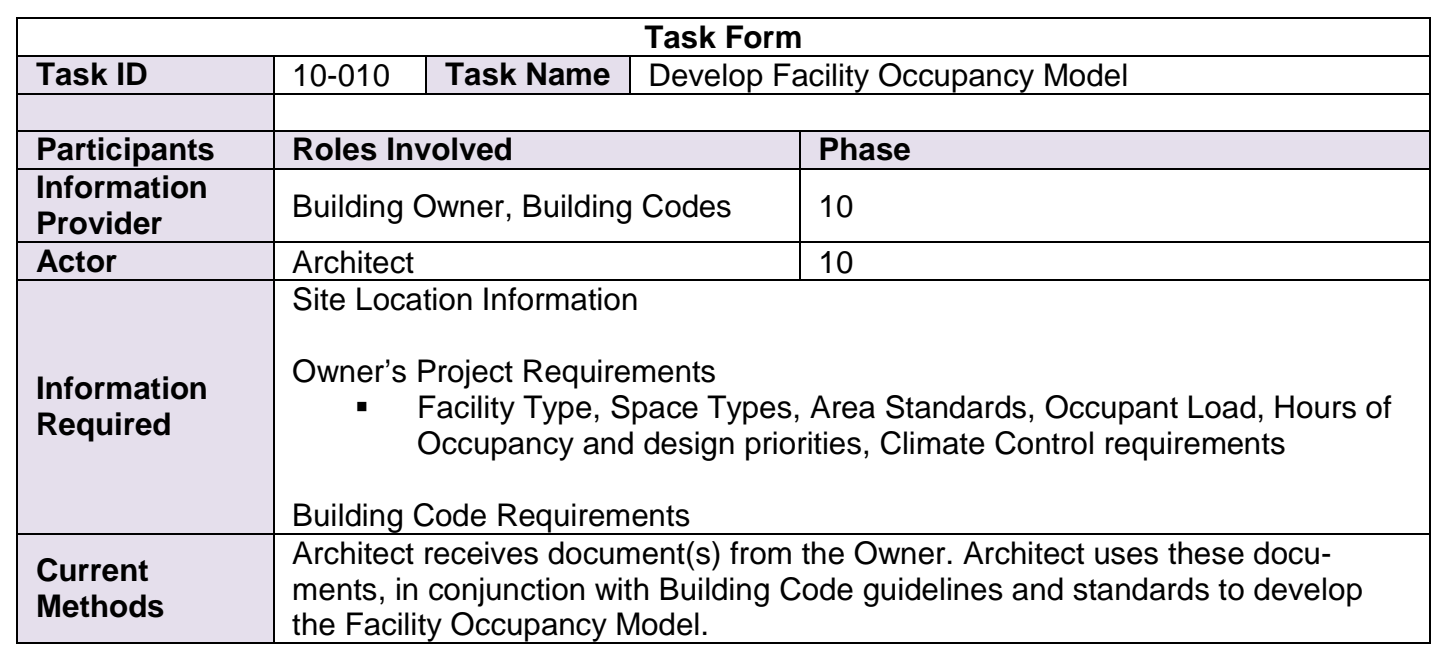

\begin{tabular}{|c|c|c|c|c|}
\hline \multicolumn{5}{|c|}{ Task Form } \\
\hline Task ID & $10-020$ & Task Name & \multicolumn{2}{|c|}{ Document Electrical Project Requirements } \\
\hline Participants & \multicolumn{3}{|c|}{ Roles Involved } & Phase \\
\hline $\begin{array}{l}\text { Information } \\
\text { Provider }\end{array}$ & \multicolumn{3}{|c|}{ Building Owner, Architect } & 10 \\
\hline Actor & \multicolumn{3}{|c|}{ Electrical Engineer } & 10 \\
\hline $\begin{array}{l}\text { Information } \\
\text { Required }\end{array}$ & \multicolumn{4}{|c|}{$\begin{array}{l}\text { Project Location (determines climate, applicable building codes, utility rate struc- } \\
\text { Occupation Factors: number of occupants, hours of occupancy, occupancy type } \\
\text { Cost Factors: level of finishes } \\
\text { Architectural Factors: size of building (area), number of floors, floor height } \\
\text { Building Environments: heating, cooling, central/unitary } \\
\underline{\text { Illumination Criteria: lighting level, light sources, daylighting, footcandles req'd, }} \\
\text { indoor/outdoor/site lighting } \\
\text { Mechanical Systems: pumps, chillers, fans (Power/Area for each space) }\end{array}$} \\
\hline $\begin{array}{l}\text { Current } \\
\text { Methods }\end{array}$ & \multicolumn{4}{|c|}{$\begin{array}{l}\text { Determine system types for consideration } \\
\text { Determine scope of major HVAC equipment } \\
\text { Determine power density at building scope }\end{array}$} \\
\hline
\end{tabular}




\begin{tabular}{|c|c|c|}
\hline \multicolumn{3}{|r|}{ Task Form } \\
\hline Task ID & Task Name & Propose Electrical Equipment Room Requirements \\
\hline Participants & Roles Involved & Phase \\
\hline $\begin{array}{l}\text { Information } \\
\text { Provider }\end{array}$ & Electrical Engineer & 10 \\
\hline Actor & Architect & 10 \\
\hline $\begin{array}{l}\text { Information } \\
\text { Required }\end{array}$ & \multicolumn{2}{|l|}{ Equipment list } \\
\hline $\begin{array}{l}\text { Current } \\
\text { Methods }\end{array}$ & \multicolumn{2}{|c|}{ Verify owner's list of equipment that may impact electrical load and location. } \\
\hline
\end{tabular}

\begin{tabular}{|c|c|c|c|}
\hline \multicolumn{4}{|r|}{ Task Form } \\
\hline Task ID & $10-040$ & Task Name & Program Spaces, Area, and Budget \\
\hline Participants & \multicolumn{2}{|c|}{ Roles Involved } & Phase \\
\hline $\begin{array}{l}\text { Information } \\
\text { Provider }\end{array}$ & \multicolumn{2}{|c|}{ Electrical Engineer } & 10 \\
\hline Actor & \multicolumn{2}{|c|}{ Architect } & 10 \\
\hline $\begin{array}{l}\text { Information } \\
\text { Required }\end{array}$ & \multicolumn{3}{|c|}{$\begin{array}{l}\text { Electrical Equipment and required spaces (Distribution Board: Footprint Area, } \\
\text { Access Area }\end{array}$} \\
\hline $\begin{array}{l}\text { Current } \\
\text { Methods }\end{array}$ & \multicolumn{3}{|c|}{$\begin{array}{l}\text { Program spaces according to size and proximity requirements. } \\
\text { Verify space sizes and ideal shapes of rooms with electrical service provider, } \\
\text { including equipment sizes and clearances around and between equipment. }\end{array}$} \\
\hline
\end{tabular}

\begin{tabular}{|c|c|c|c|}
\hline \multicolumn{4}{|r|}{ Task Form } \\
\hline Task ID & $10-050$ & Task Name & Coordinate Development of Concept Design \\
\hline Participants & \multicolumn{2}{|c|}{ Roles Involved } & Phase \\
\hline $\begin{array}{l}\text { Information } \\
\text { Provider }\end{array}$ & \multicolumn{2}{|c|}{ Electrical Engineer } & 10 \\
\hline Actor & \multicolumn{2}{|c|}{ Architect } & 10 \\
\hline $\begin{array}{l}\text { Information } \\
\text { Required }\end{array}$ & \multicolumn{3}{|c|}{ Space types, areas, proximity requirements (e.g., external utility hookup) } \\
\hline $\begin{array}{l}\text { Current } \\
\text { Methods }\end{array}$ & \multicolumn{3}{|c|}{ Review and modify spaces and areas per service provider and consultant input. } \\
\hline
\end{tabular}




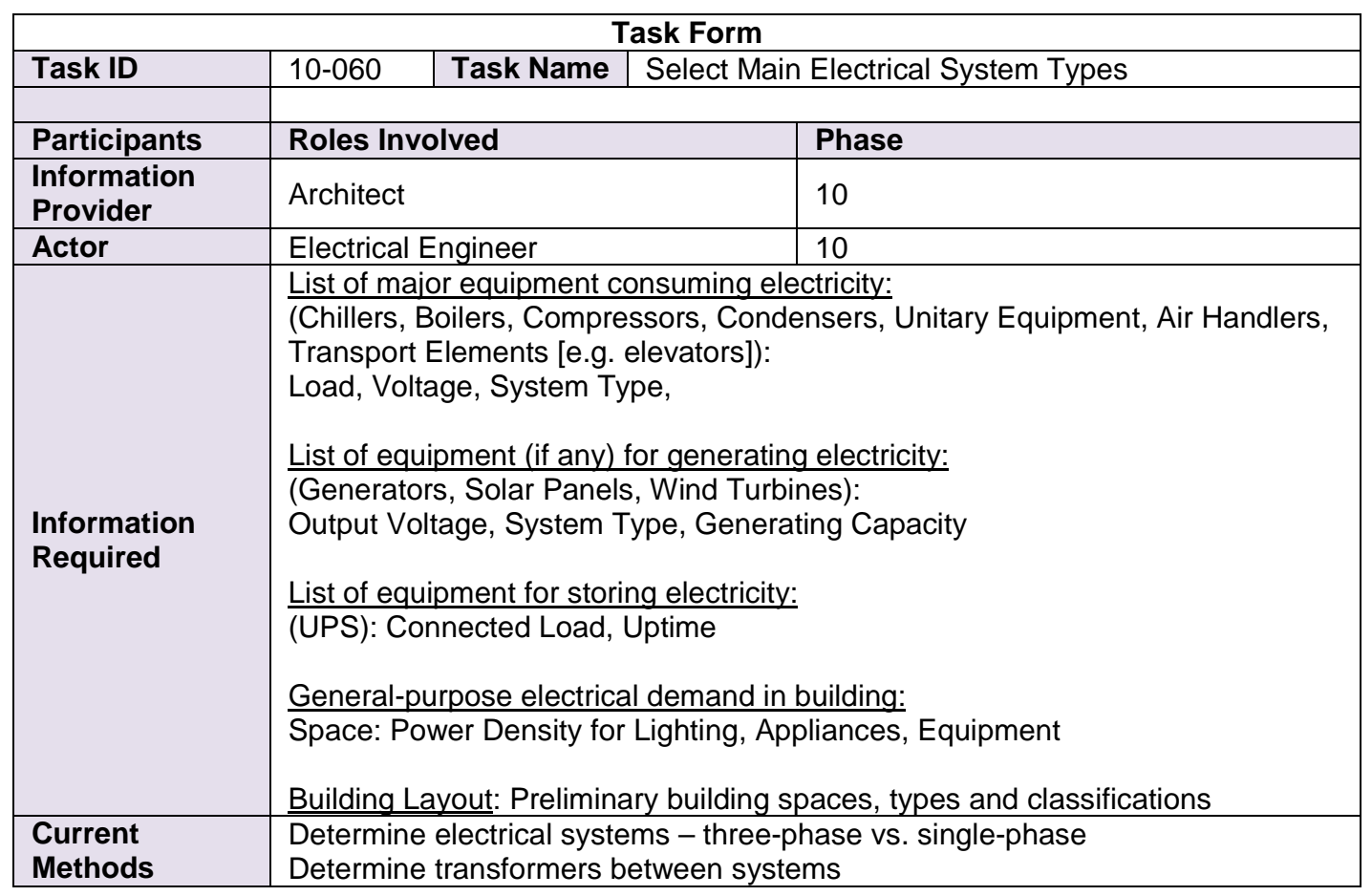

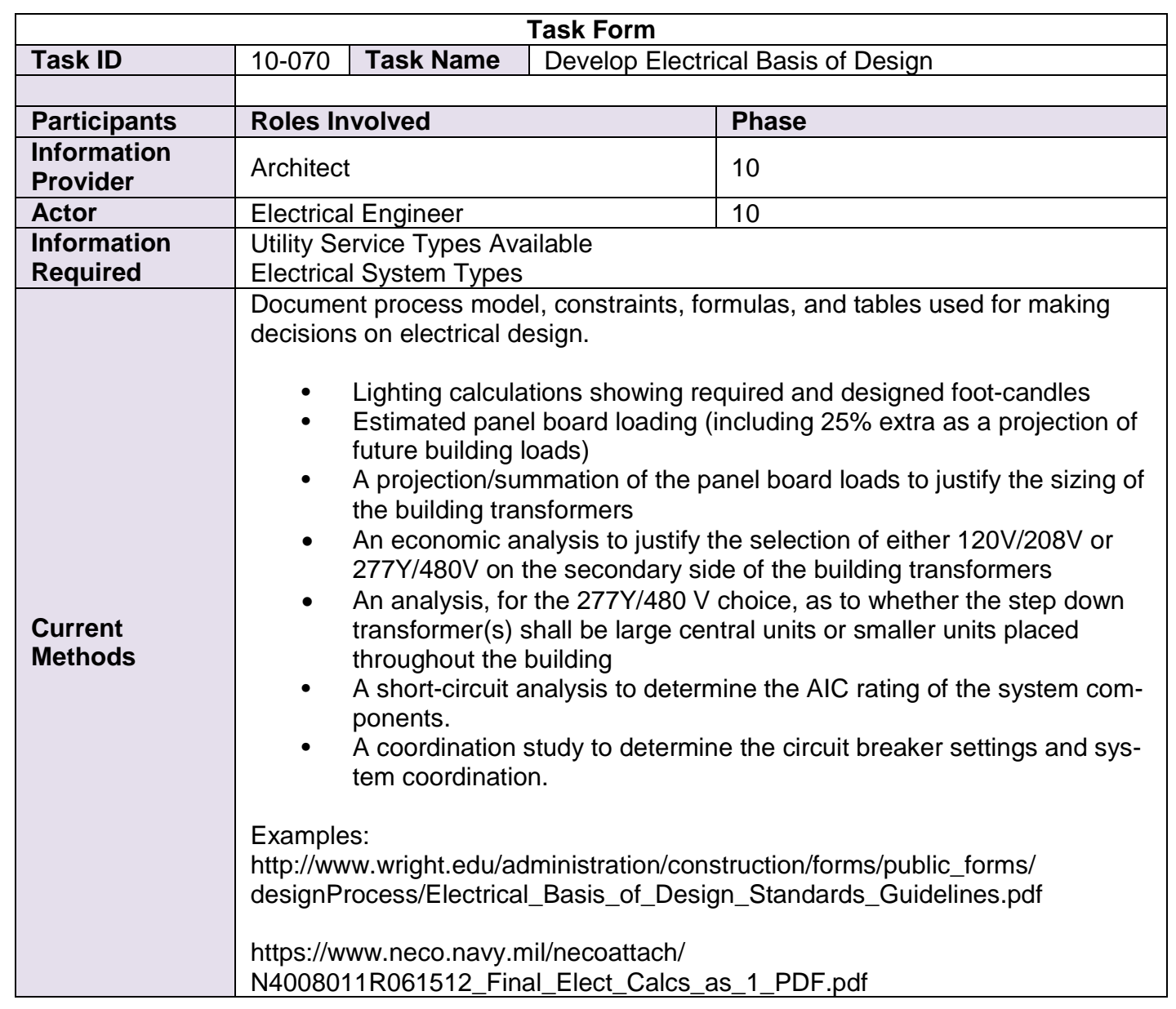




\begin{tabular}{|l|l|l|l|}
\hline \multicolumn{3}{|c|}{ Task Form } \\
\hline Task ID & $10-080$ & Task Name & Determine Source of Supply \\
\hline Participants & Roles Involved & Phase \\
\hline $\begin{array}{l}\text { Information } \\
\text { Provider }\end{array}$ & Electrical Engineer & 10 \\
\hline Actor & Electrical Engineer & 10 \\
\hline & $\begin{array}{l}\text { Utility Rate Structures, Processes, Approvals: } \\
\text { Pate structures for each system type, time intervals, and usage. }\end{array}$ \\
$\begin{array}{l}\text { Information } \\
\text { Required }\end{array}$ & $\quad$ Approval requirements. \\
& $\begin{array}{l}\text { Example: } \\
\text { Www.lipower.org/pdfs/commercial/redbook/redbook.pdf }\end{array}$ \\
\hline $\begin{array}{l}\text { Current } \\
\text { Methods }\end{array}$ & Perform economic analysis to justify selection of electrical supply source(s). \\
\hline
\end{tabular}

\begin{tabular}{|c|c|c|c|}
\hline \multicolumn{4}{|r|}{ Task Form } \\
\hline Task ID & $10-090$ & Task Name & Propose Electrical Space Requirements \\
\hline Participants & \multicolumn{2}{|c|}{ Roles Involved } & Phase \\
\hline $\begin{array}{l}\text { Information } \\
\text { Provider }\end{array}$ & \multicolumn{2}{|c|}{ Electrical Engineer } & 10 \\
\hline Actor & \multicolumn{2}{|c|}{ Architect } & 10 \\
\hline $\begin{array}{l}\text { Information } \\
\text { Required }\end{array}$ & \multicolumn{3}{|c|}{$\begin{array}{l}\text { List of major equipment for consuming, generating, transforming, and storing } \\
\text { electricity. }\end{array}$} \\
\hline $\begin{array}{l}\text { Current } \\
\text { Methods }\end{array}$ & \multicolumn{3}{|c|}{$\begin{array}{l}\text { Estimate and verify additional voltage requirements (e.g., annual, seasonal or } \\
\text { unusual circumstances), including electrical back-up generators, per code and } \\
\text { ordinances. Re-size room accordingly. }\end{array}$} \\
\hline
\end{tabular}

\begin{tabular}{|c|c|c|c|}
\hline \multicolumn{4}{|r|}{ Task Form } \\
\hline Task ID & $\begin{array}{l}10- \\
100\end{array}$ & $\begin{array}{l}\text { Task } \\
\text { Name }\end{array}$ & Estimate Energy Performance \\
\hline Participants & \multicolumn{2}{|c|}{ Roles Involved } & Phase \\
\hline $\begin{array}{l}\text { Information } \\
\text { Provider }\end{array}$ & \multicolumn{2}{|c|}{ Electrical Engineer } & 10 \\
\hline Actor & \multicolumn{2}{|c|}{ Architect } & 10 \\
\hline $\begin{array}{l}\text { Information } \\
\text { Required }\end{array}$ & \multicolumn{3}{|c|}{ Operating Costs (based on utility rate structures) } \\
\hline $\begin{array}{l}\text { Current } \\
\text { Methods }\end{array}$ & \multicolumn{3}{|c|}{$\begin{array}{l}\text { Calculate operating costs at system level based on current rate structures from } \\
\text { utility. }\end{array}$} \\
\hline
\end{tabular}




\begin{tabular}{|c|c|c|c|c|}
\hline \multicolumn{5}{|c|}{ Task Form } \\
\hline Task ID & $\begin{array}{l}10- \\
110\end{array}$ & $\begin{array}{l}\text { Task } \\
\text { Name }\end{array}$ & \multicolumn{2}{|c|}{ Document Concept Design \& Estimated Costs } \\
\hline Participants & \multicolumn{3}{|c|}{ Roles Involved } & Phase \\
\hline $\begin{array}{l}\text { Information } \\
\text { Provider }\end{array}$ & \multicolumn{3}{|c|}{$\begin{array}{l}\text { Electrical Engineer, Cost Estimator } \\
\text { (or GC in negotiated contractor } \\
\text { situation) }\end{array}$} & 10 \\
\hline Actor & \multicolumn{3}{|c|}{ Architect } & 10 \\
\hline $\begin{array}{l}\text { Information } \\
\text { Required }\end{array}$ & \multicolumn{4}{|c|}{$\begin{array}{l}\text { Space Requirements: } \\
\text { Space: area, relation to other spaces, exterior requirements } \\
\text { Mechanical Requirements: } \\
\text { Ventilation, thermal loads, fuels } \\
\text { Structural Requirements: } \\
\text { Weight } \\
\text { Construction Requirements (primarily for existing construction): } \\
\text { Installation Method, Clearances } \\
\text { Construction Costs for each system: } \\
\text { Cystems: Count } \\
\text { Operating Costs (based on systems): } \\
\text { Energy performance of proposed electrical systems, Performance History (power } \\
\text { usage time series at hourly intervals for year) }\end{array}$} \\
\hline $\begin{array}{l}\text { Current } \\
\text { Methods }\end{array}$ & \multicolumn{4}{|c|}{$\begin{array}{l}\text { Calculate construction costs at system level. } \\
\text { Calculate operating costs at system level. }\end{array}$} \\
\hline
\end{tabular}

\subsubsection{Schematic Design Phase electrical system design}

The Schematic Design Phase consists of the following tasks, shown diagrammatically in Figure 2. 
Figure 2. Process diagram for Schematic Design Phase electrical system design.

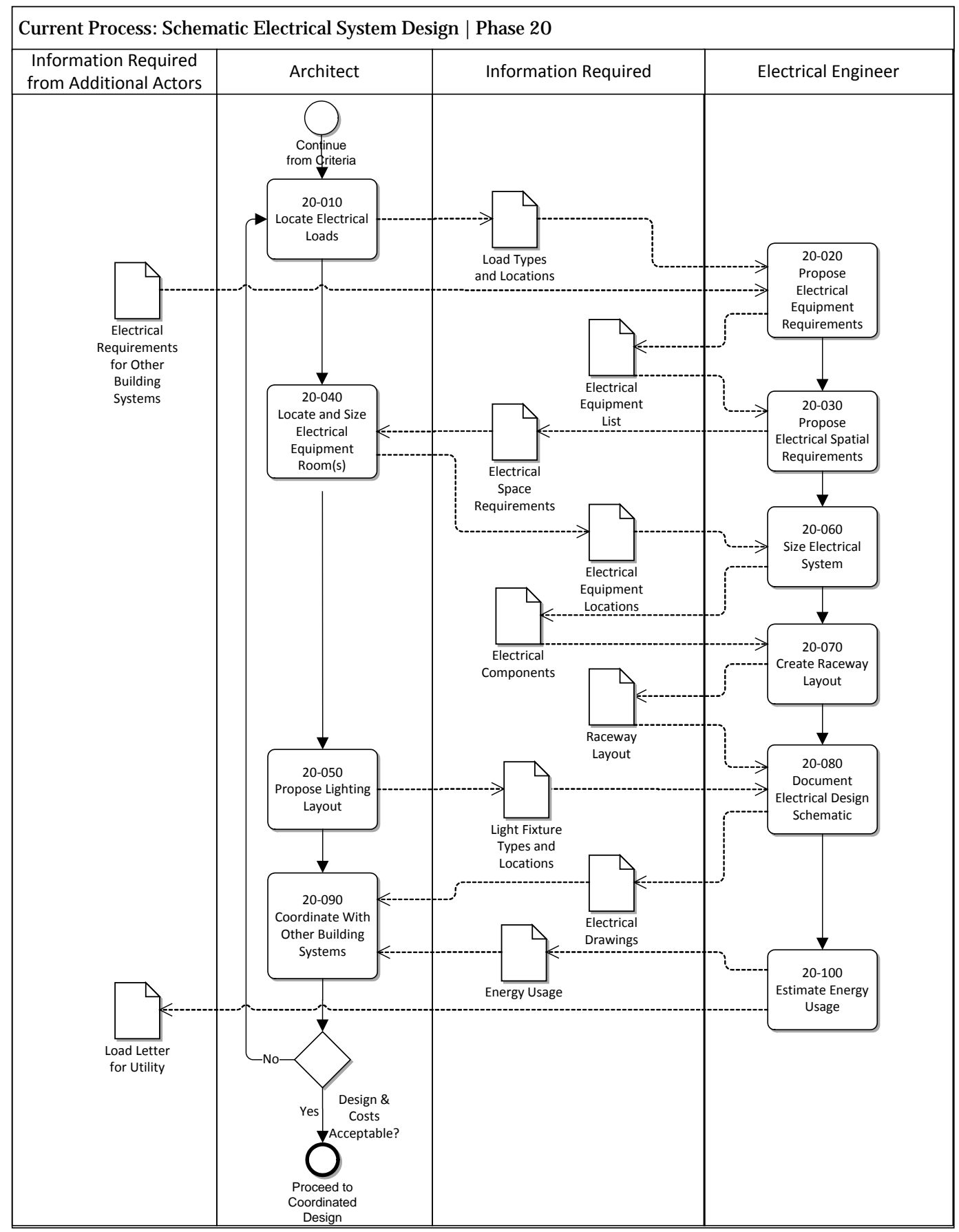




\begin{tabular}{|c|c|c|c|}
\hline \multicolumn{4}{|r|}{ Task Form } \\
\hline Task ID & $20-010$ & Task Name & Locate Electrical Loads \\
\hline Participants & \multicolumn{2}{|c|}{ Roles Involved } & Phase \\
\hline $\begin{array}{l}\text { Information } \\
\text { Provider }\end{array}$ & \multicolumn{2}{|c|}{ Electrical Engineer } & 10 \\
\hline Actor & \multicolumn{2}{|c|}{ Architect } & 20 \\
\hline $\begin{array}{l}\text { Information } \\
\text { Required }\end{array}$ & \multicolumn{3}{|c|}{$\begin{array}{l}\text { Preliminary Schedule of Electrical Load Types } \\
\text { Light fixtures, outlets, other devices consuming electricity (e.g., Unitary } \\
\text { Equipment) }\end{array}$} \\
\hline $\begin{array}{l}\text { Current } \\
\text { Methods }\end{array}$ & \multicolumn{3}{|c|}{$\begin{array}{l}\text { Architect uses the recommendations and preliminary schedule from the } \\
\text { Electrical Engineer to indicate locations of major electrical loads in the initial } \\
\text { schematic plans. }\end{array}$} \\
\hline
\end{tabular}

\begin{tabular}{|c|c|c|c|}
\hline \multicolumn{4}{|r|}{ Task Form } \\
\hline Task ID & $20-020$ & Task Name & Propose Electrical Equipment Requirements \\
\hline Participants & \multicolumn{2}{|c|}{ Roles Involved } & Phase \\
\hline $\begin{array}{l}\text { Information } \\
\text { Provider }\end{array}$ & \multicolumn{2}{|c|}{ Architect } & 20 \\
\hline Actor & \multicolumn{2}{|c|}{ Electrical Engineer } & 20 \\
\hline $\begin{array}{l}\text { Information } \\
\text { Required }\end{array}$ & \multicolumn{3}{|c|}{$\begin{array}{l}\text { Electrical requirements for all building systems } \\
\text { Load Types and Locations } \\
\text { - } \quad \text { Space: Type, Lighting Power Density, Appliance Power Density } \\
\text { - } \quad \text { Zone: Light fixtures within zones } \\
\text { - } \quad \text { Onitary Equipment: Unitary A/C locations within spaces } \\
\text { - } \quad \text { Provision for Voids: Locations for raceways } \\
\end{array}$} \\
\hline $\begin{array}{l}\text { Current } \\
\text { Methods }\end{array}$ & \multicolumn{3}{|c|}{$\begin{array}{l}\text { Generate One Line Diagram } \\
\text { http://en.wikipedia.org/wiki/One-line_diagram } \\
\text { Determine process for acquiring electrical equipment (e.g., design assist) and } \\
\text { verify that the process is acceptable to all participating parties } \\
\text { Determine connected load and demand load for each space } \\
\text { Determine diversity coefficients } \\
\text { Determine circuits } \\
\text { Determine loads at distribution points } \\
\text { Select equipment (or candidates) at each occurrence }\end{array}$} \\
\hline
\end{tabular}

\begin{tabular}{|c|c|c|c|}
\hline \multicolumn{4}{|r|}{ Task Form } \\
\hline Task ID & $20-030$ & Task Name & Propose Electrical Spatial Requirements \\
\hline Participants & \multicolumn{2}{|c|}{ Roles Involved } & Phase \\
\hline $\begin{array}{l}\text { Information } \\
\text { Provider }\end{array}$ & \multicolumn{2}{|c|}{ Electrical Engineer } & 20 \\
\hline Actor & \multicolumn{2}{|c|}{ Electrical Engineer } & 20 \\
\hline $\begin{array}{l}\text { Information } \\
\text { Required }\end{array}$ & $\begin{array}{l}\text { Electric } \\
\text { Lighting } \\
\text { Racewa }\end{array}$ & $\begin{array}{l}\text { Equipment Lis } \\
\text { ayout: Surface } \\
\text { Above ceiling }\end{array}$ & $\begin{array}{l}\text { finish, clearance } \\
\text { clearance, wall construction type (masonry/studs, etc.) }\end{array}$ \\
\hline $\begin{array}{l}\text { Current } \\
\text { Methods }\end{array}$ & \multicolumn{3}{|c|}{$\begin{array}{l}\text { Electrical Engineer uses the Electrical Equipment List and preliminary } \\
\text { architectural plans to develop proposed Electrical Space Requirements. }\end{array}$} \\
\hline
\end{tabular}




\begin{tabular}{|c|c|c|c|}
\hline \multicolumn{4}{|r|}{ Task Form } \\
\hline Task ID & $20-040$ & Task Name & Locate and Size Electrical Equipment Room(s) \\
\hline Participants & \multicolumn{2}{|c|}{ Roles Involved } & Phase \\
\hline $\begin{array}{l}\text { Information } \\
\text { Provider }\end{array}$ & \multicolumn{2}{|c|}{ Electrical Engineer } & 20 \\
\hline Actor & \multicolumn{2}{|c|}{ Architect } & 20 \\
\hline $\begin{array}{l}\text { Information } \\
\text { Required }\end{array}$ & \multicolumn{3}{|c|}{$\begin{array}{l}\text { Space: Required Area, Required Wall Lengths } \\
\text { Equipment (e.g., Distribution Board): Clearance Area } \\
\text { Cable Carrier: Location, Profile, Access Locations }\end{array}$} \\
\hline $\begin{array}{l}\text { Current } \\
\text { Methods }\end{array}$ & \multicolumn{3}{|c|}{$\begin{array}{l}\text { Verify location of service access to site. } \\
\text { Determine site lighting loads on system. } \\
\text { Reserve space for electrical use } \\
\text { Reserve site areas for electrical utilities }\end{array}$} \\
\hline
\end{tabular}

\begin{tabular}{|c|c|c|c|c|}
\hline \multicolumn{5}{|c|}{ Task Form } \\
\hline Task ID & $20-050$ & Task Name & Pro & ghting Layout \\
\hline Participants & \multicolumn{3}{|c|}{ Roles Involved } & Phase \\
\hline $\begin{array}{l}\text { Information } \\
\text { Provider }\end{array}$ & \multicolumn{3}{|c|}{ Electrical Engineer, Architect } & 20 \\
\hline Actor & \multicolumn{3}{|c|}{ Architect } & 20 \\
\hline $\begin{array}{l}\text { Information } \\
\text { Required }\end{array}$ & \multicolumn{4}{|c|}{$\begin{array}{l}\text { Space: Space Type, Lighting Density, Lighting Type } \\
\text { Light Fixture: Light Source Type, Light Emission }\end{array}$} \\
\hline $\begin{array}{l}\text { Current } \\
\text { Methods }\end{array}$ & \multicolumn{4}{|c|}{ Arrange layout of light fixtures in spaces. } \\
\hline
\end{tabular}

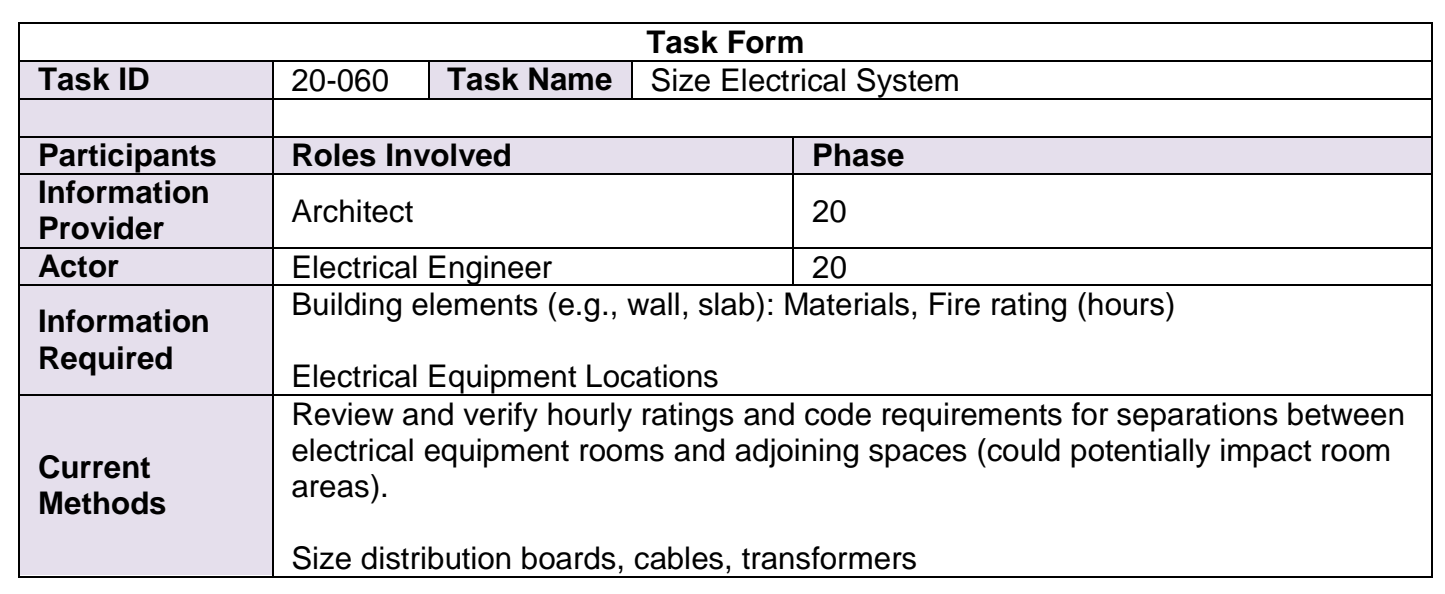




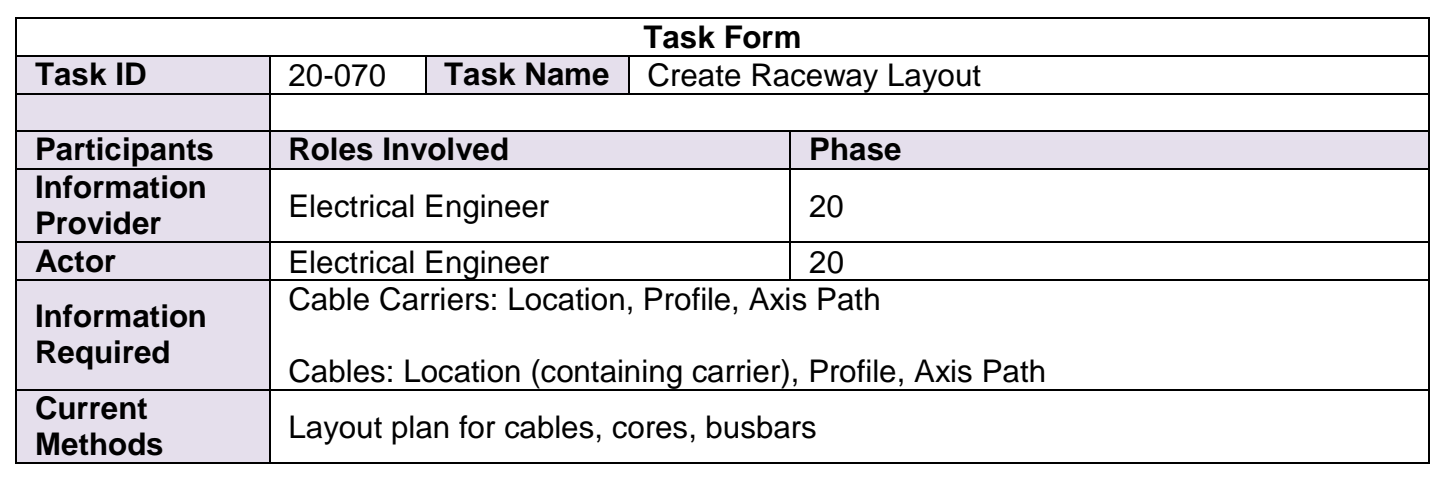

\begin{tabular}{|c|c|c|c|}
\hline \multicolumn{4}{|r|}{ Task Form } \\
\hline Task ID & $20-080$ & Task Name & Document Electrical Systems Schematic Design \\
\hline Participants & Roles I & lolved & Phase \\
\hline $\begin{array}{l}\text { Information } \\
\text { Provider }\end{array}$ & Electric & Engineer & 20 \\
\hline Actor & Electric & Engineer & 20 \\
\hline $\begin{array}{l}\text { Information } \\
\text { Required }\end{array}$ & 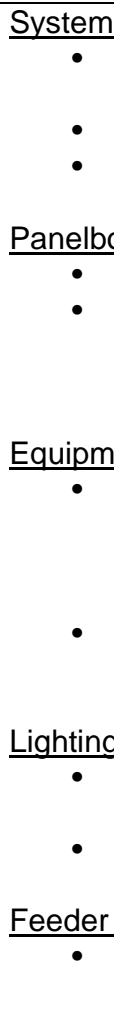 & $\begin{array}{l}\text { Systems: ID, V } \\
\text { safety) } \\
\text { Circuits: ID, Vo } \\
\text { Elements: Loce } \\
\text { rd Schedules: } \\
\text { Distribution Bo } \\
\text { Protective Dev } \\
\text { Receptacles, L } \\
\text { Protection, Circ } \\
\text { tt Schedules: } \\
\text {-quipment: Na } \\
\text { Controller Acce } \\
\text { Designation, B } \\
\text { Shase, Conduc } \\
\text { Generation Eq } \\
\text { Type, Fuel Typ } \\
\text { ixture Schedu } \\
\text { ight Fixture: T } \\
\text { Natts Per Fixt } \\
\text {-amp: Count, F } \\
\text { chedules: } \\
\text { Cable Segmen } \\
\text { f Installation, }\end{array}$ & $\begin{array}{l}\text { Itage, Type (Electrical subtype, lighting control, life } \\
\text { tage Type, Wire Size, Continuous Length, Run Length } \\
\text { tion, Mounting, Connections } \\
\text { rds: Name, Description, Capacity, Spare Capacity } \\
\text { ad: Load Name, Load KVA Lighting, Load KVA } \\
\text { ad KVA Other/Motor, Over Current Amps, Over Current } \\
\text { it Phase Balance A/B/C. } \\
\text { ne, Mark, Power, Voltage, Phase, Speed, Location, } \\
\text { anchies, Controller Type, Controller Size, Panel } \\
\text { or Ground, Conduit Size, Notes } \\
\text { ipment: Capacity, Connected Load, Transfer Switch } \\
\text { Single/Three-Phase } \\
\text { ps: } \\
\text { e, Manufacturer, Lighting Power Density (LPD) } \\
\text { ower, Lamp Code } \\
\text { Namercurrent Protection, Connected Load, Demand Load } \\
\text { Name, Cable Insulation, Size in AwG or MCM, Method }\end{array}$ \\
\hline $\begin{array}{l}\text { Current } \\
\text { Methods }\end{array}$ & $\begin{array}{l}\text { Create } \\
\text { Create }\end{array}$ & $\begin{array}{l}\text { ns from buildi } \\
\text { edules from i }\end{array}$ & $\begin{array}{l}\text { g model } \\
\text { ems and attributes }\end{array}$ \\
\hline
\end{tabular}




\begin{tabular}{|c|c|c|c|}
\hline \multicolumn{4}{|r|}{ Task Form } \\
\hline Task ID & $20-090$ & Task Name & Coordinate With Other Building Systems \\
\hline Participants & \multicolumn{2}{|c|}{ Roles Involved } & Phase \\
\hline $\begin{array}{l}\text { Information } \\
\text { Provider }\end{array}$ & \multicolumn{2}{|c|}{ Electrical Engineer } & 20 \\
\hline Actor & \multicolumn{2}{|c|}{ Architect } & 20 \\
\hline $\begin{array}{l}\text { Information } \\
\text { Required }\end{array}$ & \multicolumn{3}{|c|}{$\begin{array}{l}\text { Electrical Drawings and Specifications: Electrical plans showing equipment } \\
\text { locations as well as cable routing and connectivity } \\
\text { Electrical Schedules for equipment, fixtures, feeders, panelboards. }\end{array}$} \\
\hline $\begin{array}{l}\text { Current } \\
\text { Methods }\end{array}$ & \multicolumn{3}{|c|}{ Electrical Engineer sends the electrical drawings to the Architect. } \\
\hline
\end{tabular}

\begin{tabular}{|l|l|l|}
\hline \multicolumn{2}{|c|}{ Task Form } \\
\hline Task ID & $20-100$ & \multicolumn{2}{|c|}{ Task Name } & \multicolumn{2}{|l|}{ Estimate Energy Usage } \\
\hline & \multicolumn{2}{|c|}{ Phase } \\
\hline Participants & Roles Involved & 20 \\
\hline Information & Electrical Engineer & 20 \\
\hline Actor & Electrical Engineer & \multicolumn{1}{|c|}{ Load profile at each device consuming electricity } \\
& $\begin{array}{l}\text { Generation profile at each device generating electricity } \\
\text { (Load at hourly intervals throughout year) }\end{array}$ \\
& $\begin{array}{l}\text { Service Location } \\
\text { Total Area }\end{array}$ \\
& $\begin{array}{l}\text { Conditioned Space Area } \\
\text { Type of Heat } \\
\text { Similar Business: Name, Address, Utility Account\# } \\
\text { Information } \\
\text { Required }\end{array}$ & $\begin{array}{l}\text { Type of Service: Underground, Overhead, Service Change, Relocation, New, } \\
\text { Temporary } \\
\text { Service Characteristics: Size of Load Wires, Sets of Load Wires Per Phase, } \\
\text { Load Wire Type (AL/CU), Terminations: } \\
\text { Meterbase/C.T.Cabinet/ConnectionBox/Switchgear/Other } \\
\text { Service Size (amp): 100/150/200/300/400/600/other } \\
\text { Voltage: 1P3W-120/240, 3P4W-120/240 (<=200 amps), 3P4W-Wye-120/208, } \\
\text { 3P4W-Wye-277/480, Other } \\
\text { Electric Load Excluding Motor Load (kW): Interior Lighting, Exterior Lighting, } \\
\text { Electric Cooking, Water Heating, Dryer, Heat Pump, Heat Pump Strip Heat, } \\
\text { Computers, Receptacles, Refrigeration, Electric Heat, AC (tons): Data } \\
\text { Processing Load Only, Not Including Data Processing } \\
\text { Electric Motor Load (Except Heating and AC): Phase, Number of Motors, HP, } \\
\text { Voltage, Hours of Operation per week } \\
\text { Estimated Business Operating Time: Hours Per Week, Month Per Year } \\
\text { Meter Location Desired } \\
\text { Service Equipment Location Desired }\end{array}$ \\
\hline $\begin{array}{l}\text { Calculate connected loads and demand loads at each circuit, and for overall } \\
\text { electrical system. } \\
\text { Submit Load Letter (format provided by utility), with values specified per project. }\end{array}$ \\
\hline
\end{tabular}




\subsubsection{Coordinated Design Phase electrical system design}

The Coordinated Design Phase comprises the following tasks, shown diagrammatically in Figure 3.

Figure 3. Process diagram for Coordinated Design Phase electrical system design.

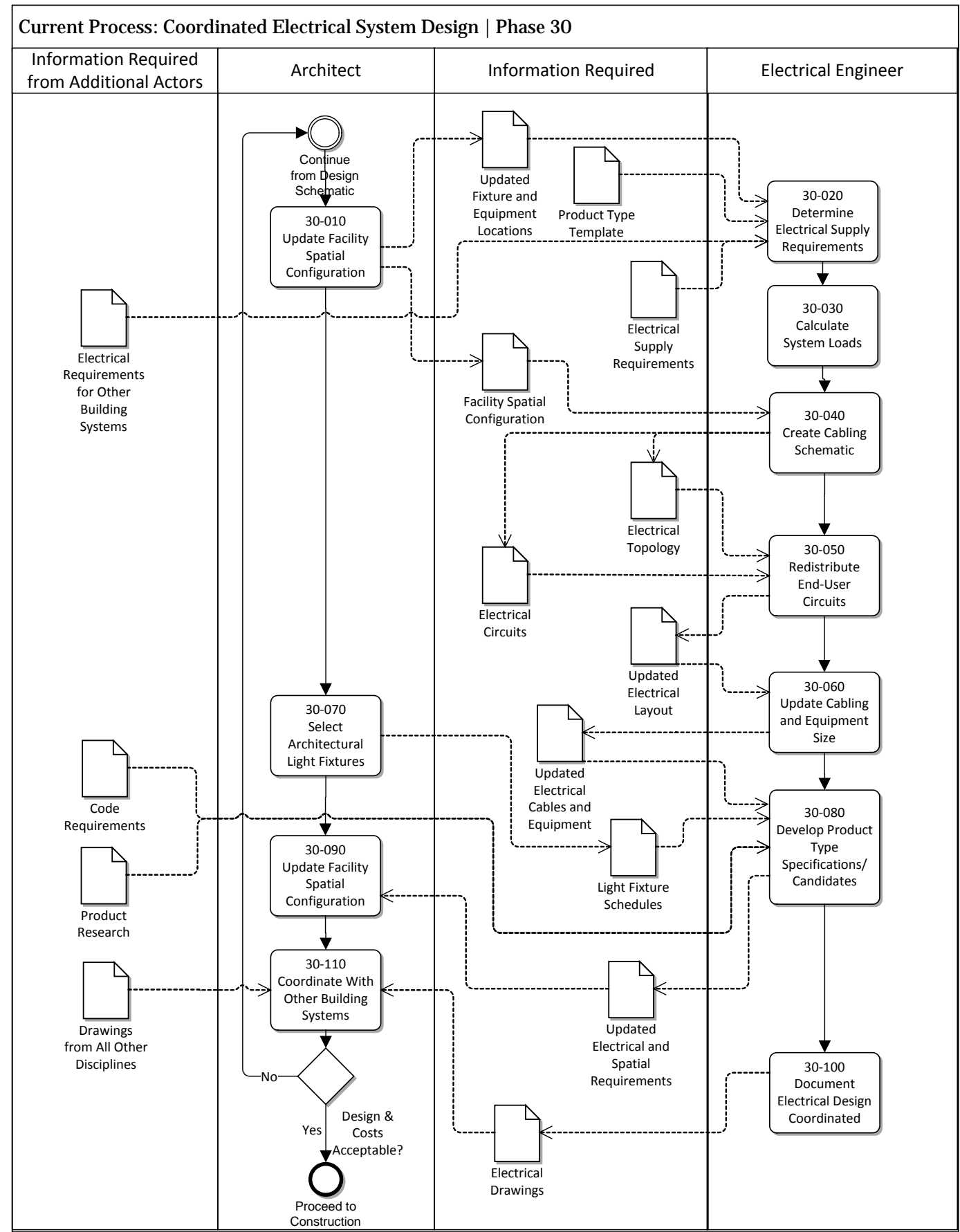




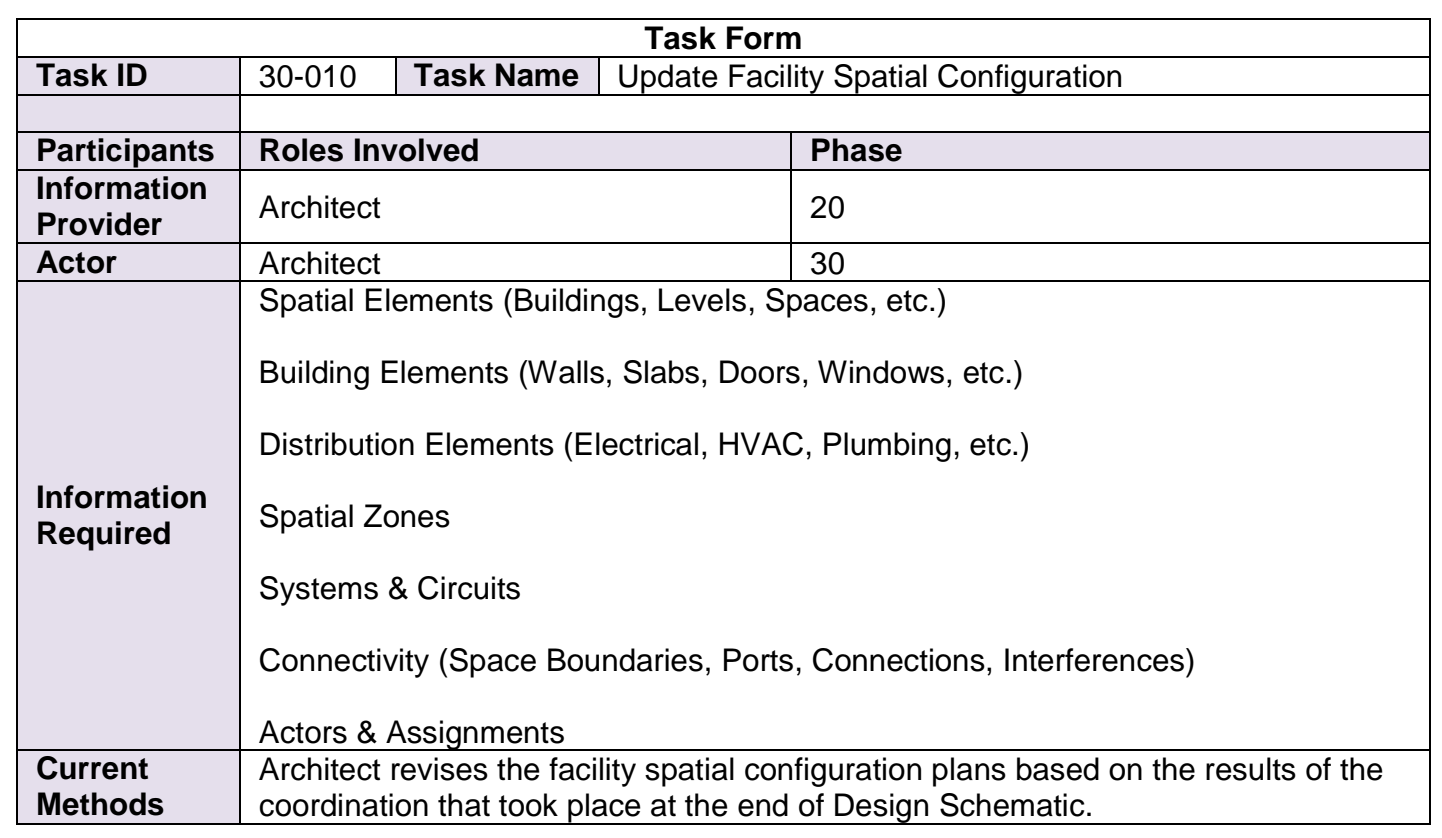

\begin{tabular}{|c|c|c|}
\hline \multicolumn{3}{|r|}{ Task Form } \\
\hline Task ID & Task Name & Determine Electrical Supply Requirements \\
\hline Participants & Roles Involved & Phase \\
\hline $\begin{array}{l}\text { Information } \\
\text { Provider }\end{array}$ & Architect & 30 \\
\hline Actor & Electrical Engineer & 30 \\
\hline $\begin{array}{l}\text { Information } \\
\text { Required }\end{array}$ & $\begin{array}{r}\text { Product Type Template: } \\
\text { Electrical system pe } \\
\text { - Cable segment } \\
\text { Schedules of Electri } \\
\text { Updated Electrical E } \\
\text { Updated Location of Ele } \\
\text { - Electrical Plan } \\
\text { System Type } \\
\text { Voltage, Phase }\end{array}$ & $\begin{array}{l}\text { formance specifications } \\
\text { size, location, amps, resistance } \\
\text { cal Fixtures and Devices } \\
\text { quipment Sizes } \\
\text { trical Fixtures \& Equipment } \\
\end{array}$ \\
\hline $\begin{array}{l}\text { Current } \\
\text { Methods }\end{array}$ & $\begin{array}{l}\text { Electrical Engineer uses } \\
\text { discipline information to } \\
\text { Select from compatible } \\
\text { [or if required, select } 3 \mathrm{c} \\
\text { Obtain owner's approval }\end{array}$ & $\begin{array}{l}\text { the Product Type Template and updated plans and other- } \\
\text { letermine total electrical supply requirements. } \\
\text { roduct types for each product occurrence. } \\
\text { mpatible product types that are suitable] }\end{array}$ \\
\hline
\end{tabular}




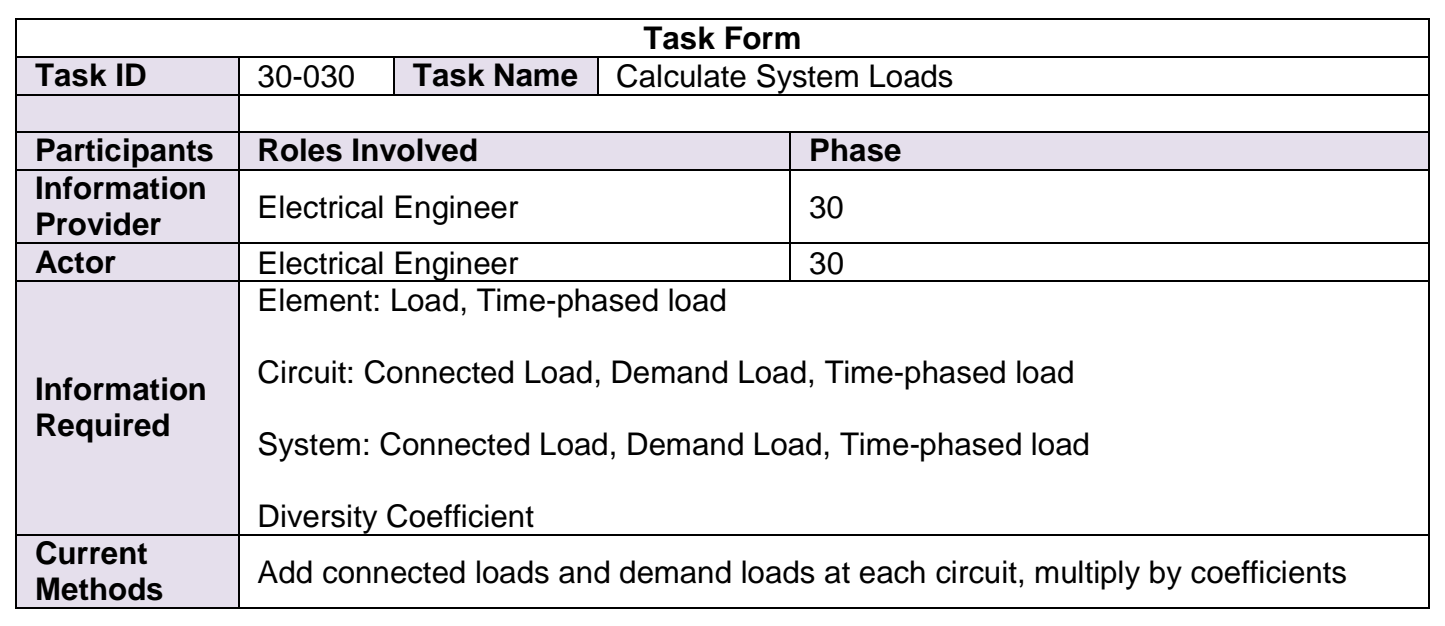

\begin{tabular}{|c|c|c|c|c|}
\hline \multicolumn{5}{|c|}{ Task Form } \\
\hline Task ID & $30-040$ & Task Name & \multicolumn{2}{|c|}{ Create Cabling Schematic } \\
\hline Participants & \multicolumn{3}{|c|}{ Roles Involved } & Phase \\
\hline $\begin{array}{l}\text { Information } \\
\text { Provider }\end{array}$ & \multicolumn{3}{|c|}{ Architect, Electrical Engineer } & 30 \\
\hline Actor & \multicolumn{3}{|c|}{ Electrical Engineer } & 30 \\
\hline $\begin{array}{l}\text { Information } \\
\text { Required }\end{array}$ & \multicolumn{4}{|c|}{$\begin{array}{ll}\text { - } & \text { Switch: Location, Lighting Load, Controls } \\
\text { - } & \text { Cable Location, Appliance Load } \\
& \text { (EMT/ENT/MC/Rigid/etc.) } \\
\text { - } & \text { Switchgear/Panels } \\
\text { - Junction Box: Location (note: may not be at this level of detail) } \\
\text { - Life Safety devices / control panels: Location } \\
\text { - } \quad \text { Lighting: Location } \\
\text { - Generating Equation } \\
\text { Location }\end{array}$} \\
\hline $\begin{array}{l}\text { Current } \\
\text { Methods }\end{array}$ & \multicolumn{4}{|c|}{ Layout Raceways, Circuits, Distribution Equipment } \\
\hline
\end{tabular}

\begin{tabular}{|c|c|c|}
\hline \multicolumn{3}{|r|}{ Task Form } \\
\hline Task ID & Task Name & Redistribute End User Circuits \\
\hline Participants & Roles Involved & Phase \\
\hline $\begin{array}{l}\text { Information } \\
\text { Provider }\end{array}$ & Electrical Engineer & 30 \\
\hline Actor & Electrical Engineer & 30 \\
\hline $\begin{array}{l}\text { Information } \\
\text { Required }\end{array}$ & $\begin{array}{l}\text { Electrical Topology } \\
\text { Electrical Circuits: Capa } \\
\text { Range, Future Expansic }\end{array}$ & ity, Connected Load, Demand Load, Desired Load Factor \\
\hline $\begin{array}{l}\text { Current } \\
\text { Methods }\end{array}$ & $\begin{array}{l}\text { For circuits with loads al } \\
\text { For circuits with loads b }\end{array}$ & $\begin{array}{l}\text { ove desired factor, split into separate circuits. } \\
\text { low minimum factor, combine circuits }\end{array}$ \\
\hline
\end{tabular}




\begin{tabular}{|c|c|c|c|}
\hline \multicolumn{4}{|r|}{ Task Form } \\
\hline Task ID & $30-060$ & Task Name & Update Cabling and Equipment Size \\
\hline Participants & Roles I & olved & Phase \\
\hline $\begin{array}{l}\text { Information } \\
\text { Provider }\end{array}$ & Electric & Engineer & 30 \\
\hline Actor & Electric & Engineer & 30 \\
\hline $\begin{array}{l}\text { Information } \\
\text { Required }\end{array}$ & $\begin{array}{l}\text { Update } \\
\text { Electric } \\
\text { Range, }\end{array}$ & $\begin{array}{l}\text { Circuits: Capa } \\
\text { Iture Expansic }\end{array}$ & ity, Connected Load, Demand Load, Desired Load Factor \\
\hline $\begin{array}{l}\text { Current } \\
\text { Methods }\end{array}$ & $\begin{array}{l}\text { Electric } \\
\text { sizes. }\end{array}$ & Engineer upda & es the schedules of raceways, cables, and equipment \\
\hline
\end{tabular}

\begin{tabular}{|c|c|c|c|c|}
\hline \multicolumn{5}{|c|}{ Task Form } \\
\hline Task ID & $30-070$ & Task Name & \multicolumn{2}{|c|}{ Select Architectural Light Fixtures } \\
\hline Participants & \multicolumn{3}{|c|}{ Roles Involved } & Phase \\
\hline $\begin{array}{l}\text { Information } \\
\text { Provider }\end{array}$ & \multicolumn{3}{|c|}{ Architect, Electrical Engineer } & 30 \\
\hline Actor & \multicolumn{3}{|c|}{ Architect } & 30 \\
\hline $\begin{array}{l}\text { Information } \\
\text { Required }\end{array}$ & \multicolumn{4}{|c|}{ Light Fixture: specific type, or assignment of 3 approved types } \\
\hline $\begin{array}{l}\text { Current } \\
\text { Methods }\end{array}$ & \multicolumn{4}{|c|}{ Select suitable type(s) from vendor catalogs } \\
\hline
\end{tabular}

\begin{tabular}{|c|c|c|c|c|}
\hline \multicolumn{5}{|c|}{ Task Form } \\
\hline Task ID & $30-080$ & Task Name & \multicolumn{2}{|c|}{ Develop Product Type Specifications/Candidates } \\
\hline Participants & \multicolumn{3}{|c|}{ Roles Involved } & Phase \\
\hline $\begin{array}{l}\text { Information } \\
\text { Provider }\end{array}$ & \multicolumn{3}{|c|}{ Electrical Engineer, Architect } & 30 \\
\hline Actor & \multicolumn{3}{|c|}{ Electrical Engineer } & 30 \\
\hline $\begin{array}{l}\text { Information } \\
\text { Required }\end{array}$ & \multicolumn{4}{|c|}{ Light fixture schedules } \\
\hline $\begin{array}{l}\text { Current } \\
\text { Methods }\end{array}$ & \multicolumn{4}{|c|}{ Resize to meet capacity, selecting alternate product types that fit requirements. } \\
\hline
\end{tabular}

\begin{tabular}{|c|c|c|c|c|}
\hline \multicolumn{5}{|c|}{ Task Form } \\
\hline Task ID & $30-090$ & Task Name & \multicolumn{2}{|c|}{ Update Facility Spatial Configuration } \\
\hline Participants & \multicolumn{3}{|c|}{ Roles Involved } & Phase \\
\hline $\begin{array}{l}\text { Information } \\
\text { Provider }\end{array}$ & \multicolumn{3}{|c|}{ Electrical Engineer, Architect } & 30 \\
\hline Actor & \multicolumn{3}{|c|}{ Architect } & 30 \\
\hline $\begin{array}{l}\text { Information } \\
\text { Required }\end{array}$ & \multicolumn{4}{|c|}{$\begin{array}{l}\text { Updated electrical layout } \\
\text { - Electrical Plan(s) - Fixtures, Equipment, Cable routing, distribution } \\
\text { sources }\end{array}$} \\
\hline $\begin{array}{l}\text { Current } \\
\text { Methods }\end{array}$ & \multicolumn{4}{|c|}{$\begin{array}{l}\text { Architect revises the facility spatial configuration plans based on the updated } \\
\text { electrical layout and spatial requirements provided by the Electrical Engineer. }\end{array}$} \\
\hline
\end{tabular}




\begin{tabular}{|c|c|c|c|c|}
\hline \multicolumn{5}{|c|}{ Task Form } \\
\hline Task ID & $30-100$ & Task Name & \multicolumn{2}{|c|}{ Document Electrical Design Coordinated } \\
\hline Participants & \multicolumn{3}{|c|}{ Roles Involved } & Phase \\
\hline $\begin{array}{l}\text { Information } \\
\text { Provider }\end{array}$ & \multicolumn{3}{|c|}{ Electrical Engineer } & 30 \\
\hline Actor & \multicolumn{3}{|c|}{ Electrical Engineer } & 30 \\
\hline $\begin{array}{l}\text { Information } \\
\text { Required }\end{array}$ & \multicolumn{4}{|c|}{ 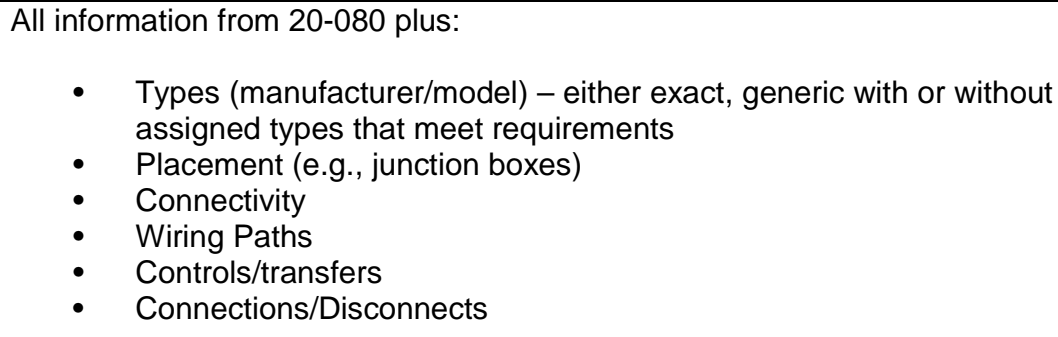 } \\
\hline $\begin{array}{l}\text { Current } \\
\text { Methods }\end{array}$ & \multicolumn{4}{|c|}{$\begin{array}{l}\text { Create plans with detail on elements. } \\
\text { Create schedules based on elements and acceptable product types. }\end{array}$} \\
\hline
\end{tabular}

\begin{tabular}{|c|c|c|c|c|}
\hline \multicolumn{5}{|c|}{ Task Form } \\
\hline Task ID & $30-110$ & Task Name & \multicolumn{2}{|c|}{ Coordinate With Other Building Systems } \\
\hline Participants & \multicolumn{3}{|c|}{ Roles Involved } & Phase \\
\hline $\begin{array}{l}\text { Information } \\
\text { Provider }\end{array}$ & \multicolumn{3}{|c|}{ Electrical Engineer } & 30 \\
\hline Actor & \multicolumn{3}{|c|}{ Architect } & 30 \\
\hline $\begin{array}{l}\text { Information } \\
\text { Required }\end{array}$ & \multicolumn{4}{|c|}{$\begin{array}{l}\text { Updated Electrical drawings showing physical size and location of all elements in } \\
\text { the electrical system } \\
\text { Final Electrical Specifications }\end{array}$} \\
\hline $\begin{array}{l}\text { Current } \\
\text { Methods }\end{array}$ & \multicolumn{4}{|c|}{$\begin{array}{l}\text { Electrical Engineer sends the electrical drawings to the architect. Architect } \\
\text { incorporated the information into the design. }\end{array}$} \\
\hline
\end{tabular}




\section{Fundamental Concepts}

\subsection{Overview}

This chapter documents common concepts of information modeling applied to various object types found in data exchanges. Each individual concept template may also be referred to as a functional part, and describes a graph of object classes and attributes. Such templates are further refined at each applicable object class to indicate specific values or types that may be used. For a complete presentation of the MVD, including IFC instance diagrams and tables indicating how the concepts are used by entities for exchanges, see http://docs.buildingsmartalliance.org/MVD SPARKIE.

\subsection{Concept templates}

Various concept templates have been introduced in this specification, and existing concept templates have been adapted from the IFC4 specification of BuildingSmart International (www.buildingsmart-tech.org).

NOTE: This specification is also available in HTML and MVDXML form, where the online specification contains additional content including instantiation diagrams and exchange requirements tables.

This specification consists of a schema defining data types, along with common concepts indicating use of data types for particular scenarios. This chapter defines such common concepts, which are applied at entities having specific use. Such concepts also form the basis of Model View Definitions, which are supplementary specifications that adapt the scope and rules of this schema for targeted domains within the building industry.

Each concept template defines a graph of entities and attributes, with constraints and parameters set for particular attributes and instance types. Various entities within this schema reference such concept templates and adapt them for particular use according to parameters. For example, the 'Ports' concept template defines distribution system connectivity for mechanical, electrical, and plumbing systems and a pipe segment defines an application of the 'Ports' concept, having one port as an inlet and another as an outlet. 


\subsubsection{Roots}

All entities having semantic significance derive from IfcRoot, where instances are identifiable within a data set using a compressed globally unique identifier (IFC-GUID). This identifier must never change during the lifetime of an object, which allows data to be merged, versioned, or referenced from other locations.

Resource-level instances (not deriving from IfcRoot) do not have any identity, such that two instances having identical state are considered equal. For example, if an object has coordinates described by an IfcCartesianPoint instance, another object with the same coordinates may have a separate instance of IfcCartesianPoint or share the same instance; such difference is a matter of data storage optimization and does not imply any semantic relationship. This also implies that non-rooted instances may only exist if referenced by at least one rooted instance through either a direct attribute or inverse attribute, or following a chain of attribute references on instances.

The distinction between rooted and non-rooted (resource-level) entities achieves several goals:

- File size may be reduced by interning (sharing) non-rooted data instances;

- Database retrieval may be more efficient by storing non-rooted data local to rooted data instances;

- Storage size may be reduced by avoiding IFC-GUID storage for items not requiring direct retrieval;

- Comparisons of differences may be done at a higher level where the context of such change is apparent;

- Implementations may treat non-rooted data instances as immutable for efficiency or simplified usage.

\subsubsection{Identity}

An object needs to be identifiable for accurate processing by both human and automated processes. Identification may be through several attributes such as Identification, Name, Description or GUID. The GUID is compressed for the purpose of being exchanged within an IFC data set - the compressed GUID is referred to as "IFC-GUID". While the IFC-GUID is normally generated automatically and has to be persistent, the Identifica- 
tion may relate to other informal registers but should be unique within the set of objects of the same type. The Name and Description should allow any object to be identified in the context of the project or facility being modeled.

Various objects may have additional identifications that may be humanreadable and/ or may be structured through classification association.

Various file formats may use additional identifications of instances for serialization purposes; however there is no requirement or guarantee for such identifications to remain the same between revisions or across applications. For example, the IFC-SPF file format lists each instance with a 64bit integer that is unique within the particular file.

\subsubsection{Project}

All files contain a single IfcProject instance indicating overall context and a directory of objects contained within.

\subsubsection{Project declaration}

The project provides a directory of objects contained within using declaration relationships

\subsection{Object type definitions}

Declaration of object types, such as element types utilized by the element occurrences within this project, within the context of the project

\subsection{Property set templates}

Declaration of property set templates, including the property templates that are used as property definitions. Such templates define the applicable properties, their names, descriptions, measure types and property type (single, enumerated, bounded list or table value).

\subsubsection{Project units}

The project context includes the definition of the default units within the IFC data set. Default units are those units that apply: 
- To all geometric representation items within the geometric representation contexts;

- To all attributes with a defined datatype indicating a measure datatype;

- To all properties and quantities with a defined datatype indicating a measure datatype and with no local unit definitions provided.

\subsubsection{Project context}

A project representation context indicates the coordinate system orientation, direction of true north, precision, and other values that apply to all geometry within a project or project library. 3.2.3 Actor

An actor is a person or organization participating in a project. Actors may fulfill one or more roles such as engineers, contractors, manufacturers, building occupants, etc.

\subsubsection{Contact}

Contact information indicates roles and addresses of people and organizations.

\subsubsection{Control}

A control is a directive to meet specified requirements such as for scope, time, and/ or cost.

\subsubsection{Cost}

Cost information is used to indicate rate structures within a cost schedule which are applicable to assigned objects.

\subsubsection{Calendar}

Calendar information is used to filter other objects to indicate time periods during which the control applies.

\subsubsection{Product}

A product is an occurrence of a physical or virtual object with finite spatial extent. 


\subsubsection{Product placement}

Product occurrences can be placed in 3D space relative to where they are contained. Placement is defined by a relative position (X, Y, Z coordinates), a horizontal reference direction, and a vertical axis direction. At the outermost level, relative directions are defined according to representation context; for example, $+\mathrm{X}$ may point east, $+\mathrm{Y}$ may point north, and $+\mathrm{Z}$ may point up.

Placement follows aggregation and containment relationships as follows:

- At the outermost level, a site is globally positioned according to latitude, longitude, and elevation;

- For spatial structures, positioning is relative to aggregation. For example, a site may aggregate multiple buildings, each building may aggregate multiple building stories, and each building story may aggregate multiple spaces;

- For building elements, positioning is relative to the containing spatial structure. For example, a building story may contain slabs, walls, columns, and beams;

- For aggregated parts, positioning is relative to aggregation. For example, a staircase may aggregate one or more stair flights;

- For feature elements, positioning is relative to the affected building element. For example, an opening element is positioned relative to the wall it voids, which in turn is positioned relative to a building story;

- For fillings, positioning is relative to the filled opening. For example, a door is positioned relative to an opening which in turn is positioned relative to a wall;

- For distribution ports, positioning is relative to the containing distribution element. For example, an air terminal may have a port connection for a duct segment or fitting;

- For distribution elements, positioning is relative to the containing spatial structure, however may be constrained by port connections. For example, an electrical junction box may fill an opening within a wall, and the junction box may contain ports for contained outlets or switches; the placement of such connected elements is constrained relative to connected port of the junction box. As another example, an air terminal may fill a ceiling covering which is placed relative to a space; the placement of a connecting duct fitting may be constrained relative to the air terminal. 
If a containing spatial structure contains a grid, then placement may also be based relative to grid coordinates.

\subsubsection{Product representation}

The shape of products may be represented in multiple ways for different purposes. Each representation has a well-known string identifier and a particular representation context. There may be multiple representation contexts to describe a shape at various levels of detail. Most building elements have a 'Body' representation which defines or approximates the physical shape and volume. In addition to physical building elements, non-physical elements may have representations such as spaces and openings.

\subsection{Axis geometry}

Elements following a path provide an 'Axis' representation indicating a line segment or any arbitrary open bounded curve. Examples of such elements include walls, beams, columns, pipes, ducts, and cables. For elements that have a material profile set association indicating cross-section, a 'Body' representation may be generated based on the axis curve and material profiles. Curve styles may indicate particular colors, line thicknesses, and dash patterns for $2 \mathrm{D}$ rendering.

\subsection{Footprint geometry}

Elements filling a boundary provide a 'Footprint' representation indicating a rectangle or any arbitrary set of outer and inner boundary curves. Examples of such elements include slabs and spaces. For elements that have a material layer set association indicating material thicknesses, a 'Body' representation may be generated based on the footprint and material layers. Fill area styles may indicate particular colors, tiles, or hatching for 2D rendering.

The representation identifier of the footprint geometric representation is:

IfcShapeRepresentation. RepresentationIdentifier $=$ 'FootPrint' 


\subsection{Surface geometry}

Elements may have a 'Surface' representation describing the outer surface of the object. Such representation may be used for hit-testing objects having part composition such as framed walls.

\subsection{Body geometry}

Elements may have a 'Body' representation describing the volumetric shape of the object. Such representation may be used for 3D rendering or quantity take-off. Geometry may be based on boundary representations describing outer faces, primitives such as spheres or cones, swept solids such as profile extrusions or revolutions, Constructive Solid Geometry (CSG) such as clippings or subtractions of other shapes, or Non-Uniform Rational B-Spline (NURBS) geometry. Surface styles may indicate particular colors, textures, and reflectance for 3D rendering.

The representation identifier of the body representation is:

IfcShapeRepresentation. RepresentationIdentifier $=$ 'Body'

\subsection{Lighting geometry}

Elements emitting light provide a 'Lighting' representation. Examples of such elements include lamps and light fixtures. Such representation may be used for 3D rendering or lighting design.

\subsection{Clearance geometry}

Elements requiring surrounding space for clearance provide a 'Clearance' representation. The reason for clearance space may be due to ventilation, maintenance, or other purpose. Examples of such elements include boilers and chillers. Such representation may be used for interference checks, where the 'Clearance' representation must not intersect with the 'Body' representation of other objects, though may intersect with the 'Clearance' representation of other objects.

\subsubsection{Site location}

The site location may be used to determine climate conditions and applicable building codes. 


\subsubsection{Building location}

The building location may indicate the address as found on a map.

\subsubsection{Grid}

Grids are used for layout according to rectangular, triangular or circular patterns.

\subsubsection{Resource}

A resource represents usage of something, having costs and environmental impacts.

\subsubsection{Resource cost}

Resources can have associated costs indicating financial costs and environmental impacts incurred according to a specified base quantity.

Each cost value may be defined using a constant amount or calculated according to specified formula.

\subsubsection{Resource quantity}

Resources may be defined according to a base quantity, where assigned tasks consume such amount of resource relative to an output quantity.

For work-based resources such as labor and equipment, quantities are based on time. For product-based resources, quantities are based on count. For material-based resources, quantities are based on volume.

\subsubsection{Resource type}

A resource type represents a template of usage of something, having cost rates and environmental impact rates.

\subsubsection{Resource cost rate}

Resource cost rates are provided for anything that may be sold in quantity, such as product models that may be ordered, or common services that may be priced by unit. 


\subsubsection{Association}

Association refers to relating objects to external information such as documents, databases, and classifications.

\subsubsection{Classification}

Objects, type objects, properties, and some resource schema entities can be further described by associating references to external sources of information. The source of information can be:

- A classification system;

- A dictionary server;

- Any external catalogue that classifies the object further;

- A service that combine the above features.

An individual item within the external source of information can be selected. It then applies the inherent meaning of the item to the object or property.

\subsubsection{Material}

Any product or product type can have associated materials indicating the physical composition of an object. Materials can have representations for surface styles indicating colors, textures, and light reflectance for 3D rendering. Materials can have representations for fill styles indicating colors, tiles, and hatch patterns for 2D rendering. Materials can have properties such as density, elasticity, thermal resistance, and others as defined in this specification. Materials can also be classified according to a referenced industry standard.

An object can be comprised of a single material or a set of materials with a particular layout. Several examples include:

- A slab may have an associated layer of concrete;

- A beam may have an associated I-Shape profile of steel;

- A door may have associated constituents for framing and glazing;

- A port may have an associated profile and/ or material flowing through it such as hot water. 
EXAMPLE: Material information can also be given at object type, defining the common material data for all occurrences of the same type. It is then accessible by the inverse IsTypedBy relationship pointing via HasAssociations and via IfcRelAssociatesMaterial.RelatingMaterial to the material information. If both are given, then the material directly assigned to object occurrence overrides the material assigned to object type.

\subsection{Material profile set}

Material profile sets are associated with elements or element types where materials are placed in cross-sections of specified dimensions following a path defined at occurrences of the type. Examples of such products are beams, columns, members, reinforcing, footings, piles, pipe segments, duct segments, and cable segments.

Material profile sets are associated by using the relationship IfcRelAssociatesMaterial having the RelatingMaterial pointing to an IfcMaterialProfileSet. The RelatedObjects either point to a single or multiple occurrences of IfcElement, or to a single or multiple IfcElementType.

EXAMPLE: Material profile sets can be provides at the IfcColumnType, defining the common material information for all occurrences of the same column type. It is then accessible by the inverse IsTypedBy relationship at IfcColumn pointing to IfcColumnType having the HasAssociations inverse relationship to IfcRelAssociatesMaterial with RelatingMaterial refering to the IfcMaterialProfileSet. If an individual material association is provide at the IfcColumn and the IfcColumnType, then the material directly assigned to IfoColumn overrides the material assigned to IfcColumnType.

\subsection{Material profile set usage}

Material profile set usage defines layout at occurrences to indicate the offset from the 'Axis' reference curve according to cardinal point, and a reference extent such as for a default column height.

\subsubsection{Definition}

Objects may be defined by having a number of properties, where such properties may be organized partially (into property sets) or fully (into templates). 


\subsubsection{Object typing}

Object occurrences can be defined by a particular object type, using the Object Typing concept. A pair of entities is defined for most semantic objects - an object occurrence entity and a corresponding object type entity.

EXAMPLE: The IfcTank is the object occurrence entity that has a corresponding IfcTankType being the object type entity.

On instance level, an object occurrence instance may have:

- Similar state as its object type instance by applying all characteristics defined at the type;

- Overridden state for particular characteristics;

- No defined object type instance.

- Characteristics defined at the object type level may include:

- Common naming and predefined type;

- Common properties within a type driven property set;

- Common geometry representations, applied as mapped representation to each occurrences;

- Common material assignments (with exception of material set usages);

- Common definition of a decomposition structure.

Many object occurrence and object type entities have an attribute named PredefinedType consisting of a specific enumeration. Such predefined type essentially provides another level of inheritance to further differentiate objects without the need for additional entities. Predefined types are not just informational; various rules apply such as applicable property sets, part composition, and distribution ports.

EXAMPLE: For scenarios of object types having part compositions, such parts may be reflected at object occurrences having separate state. For example, a wall type may define a particular arrangement of studs, a wall occurrence may reflect the same arrangement of studs, and studs within the wall occurrence may participate in specific relationships that do not exist at the type such as being connected to an electrical junction box.

The object type is attached using the IfcRelDefinesByType objectified relationship and is accessible by the IsTypedBy inverse attribute. Only a maximum one, or zero, object types can define an object occurrence. If the 
object type has aggregated elements, such objects are reflected at the object occurrence using the IfcRelDefinesByObject relationship.

\subsubsection{Property sets}

Any specialization of object can be related to multiple property set occurrences. A property set contains multiple property occurrences. The data type of property occurrence are single value, enumerated value, bounded value, table value, reference value, list value, and combination of property occurrences.

\subsubsection{Property sets for types}

For object types, property sets are defined directly.

This concept is used by entities for exchanges as shown.

\subsubsection{Property sets for performance}

For performance history, properties are in the form of time series, for tracking data at points in time.

\subsubsection{Composition}

Objects may be composed into parts to indicate levels of detail, such as a building having multiple stories, a framed wall having studs, or a task having subtasks. Composition may form a hierarchy of multiple levels, where an object must have a single parent, or if a top-level object then declared within the single project or a project library.

\subsubsection{Object aggregation}

An aggregation indicates an unordered part composition relationship between the whole structure, referred to as the "composite", and the subordinate components, referred to as the "parts". The concept of aggregation is used in various ways. Examples are:

- Aggregation is used on building elements to indicate parts such as studs within a wall;

- Aggregation is used on spatial elements to indicate a spatial structure such as a story within a building; 
- Aggregation is used on systems to indicate subsystems such as branch circuits.

Aggregation is a bi-directional relationship, the relationship from the composite to its parts is called Decomposition, and the relationship from the part to its composite is called Composition.

\subsection{Element decomposition}

Element decomposition refers to an aggregation structure where the element, representing the composite, is decomposed into parts represented by other elements.

The composite then provides, if such concepts are in scope of the Model View Definition, exclusively the following:

- Placement - the common object coordinate system to which the parts are placed relative

- By default the following constraints apply to an element being decomposed by Element Decomposition:

- Body Geometry - composite is constructed from the sum of the Body Geometry of the parts;

- The composite shall not have an own Body Geometry, body geometry is provided at the parts;

- The composite shall not have an own Material assignment, material is assigned to the parts.

\subsection{Spatial decomposition}

Spatial decomposition refers to an aggregation structure where a spatial structure of the project is decomposed into parts represented by other spatial structure elements. The spatial structure is a hierarchical tree of spatial structure elements (site, building, story, space) ultimately assigned to the project. Decomposition refers to the relationship to lower level elements (e.g., this story has spaces).

The order of spatial structure elements being included in the concept are from high to low level: IfcProject, IfcSite, IfcBuilding, IfcBuildingStorey, IfcSpace. Therefore a spatial structure element can only has parts of an element at the same or lower level. 


\subsubsection{Object nesting}

Nesting indicates an ordered arrangement relationship. Nesting is used:

- On building elements to indicate features placed in sequence such as ports.

- On control objects to indicate specification hierarchies.

- On process objects to indicate subordinate task details.

- On resource objects to indicate subordinate resource allocations.

\subsubsection{Ports}

Ports indicate possible connections to other objects according to specified system types, flow direction, and connection properties. Ports are typically connected between devices via cables, pipes, or ducts.

Ports may have placement defined indicating the position and outward orientation of the port relative to the product or product type.

Ports may have material profile sets defined indicating the flow area and connection enclosure.

\subsubsection{Assignment}

Objects may provide services to other objects, where the "assigned from" object acts upon or observes requirements of the "assigned to" object. There is a general cycle of assignments where actors (people) issue controls (such as work orders or schedules), which may result in groups (such as building systems) comprised of products (such as building elements) operated upon by processes (such as construction tasks) performed by resources (such as labor) which may in turn be fulfilled by actors (people). Requirements are established at the "to" side and are fulfilled at the "from" side, where costs, time, scope, or other metrics may be calculated in the "from-to" direction.

\subsubsection{Actor assignment}

Actors may have assignments indicating objects for which they have responsibility. An example of such assignment is a work order assigned to an organization. 


\subsubsection{Control assignment}

Controls may have assignments indicating objects that must observe the established requirements. An example of such assignment is a labor resource assigned to a calendar.

\subsubsection{Group assignment}

Group Assignment established an arbitrary collection of objects within a group. The grouping relationship does not apply any other meaning then grouping objects under some aspect. It is non-hierarchical, that is objects can be grouped into different logical groups, and it does not interfere with other relationship concepts, such as ObjectAggregation.

The Group Assignment concept establishes a given object as being the group collection for other objects. It usually implies the existence of a grouping relationship and the provision of some identity under which the group is characterized.

The group collection is handled by an instance of IfcRelAssignsToGroup, which assigns all group members to the IfcGroup being the collection.

Objects included in group as collected items are linked by IsGroupedBy pointing to IfcRelAssignsToGroup

NOTE: The IfcGroup maybe not yet have a grouping relationship established, it then identifies an empty group.

EXAMPLE: An air handler belonging to an air conditioning system is an example of such group assignment.

\subsubsection{Process assignment}

Processes may have assignments indicating resources consumed or occupied by the process. An example of such assignment is a carpenter labor resource building a wall.

\subsubsection{Resource assignment}

Resources may have assignments indicating sources available to be used. An example of such assignment is a person fulfilling a carpenter labor resource. 


\subsubsection{Product type assignment}

Product types may have assignments indicating re-usable process types for which occurrences may operate on occurrences of the product type. An example of such assignment is a task type for placing concrete in slabs on grade.

\subsubsection{Process type assignment}

Process types may have assignments indicating re-usable resource types for which occurrences may be consumed or occupied by occurrences of the process type. An example of such assignment is a concrete mixer resource type for delivering concrete.

\subsubsection{Connectivity}

Objects may participate in various connectivity relationships with other objects.

\subsubsection{Spatial structure}

Spatial structures, such as site, building, story, or spaces, may contain physical elements, including building elements, distribution elements, and furnishing elements. The containment relationship between the physical elements and the spatial structures is hierarchical, i.e. a physical element shall only be contained within a single spatial structure.

EXAMPLE: An IfcBeam is placed within the spatial hierarchy using the objectified relationship IfcRelContainedInSpatialStructure, referring to it by its inverse attribute SELF IfcElement.ContainedInStructure. Subtypes of IfcSpatialStructureElement are valid spatial containers, with IfcBuildingStorey being the default container.

The spatial containment relationship, together with the Spatial decomposition relationship, being hierarchical as well, establishes the hiearchical project tree structure in a building information model.

EXAMPLE: The IfcBuildingStorey that logically contains the IfcBeam decomposes the IfcBuilding using the IfcRelAggregates relationship. Therefore the IfcBeam is also indirectly contained in the building. 


\subsection{Spatial containment}

The Spatial Containment concept defines the relationship of physical elements, such as building elements, distribution elements, or furnishing elements as being contained within a spatial structure element.

\subsection{Space coverings}

Spaces may have related coverings for floorings, ceilings, and wall coverings. Such relationship provides for parametric use of building materials where such elements may automatically adapt to changes in the size, layout, and openings for the space.

\subsubsection{Element connectivity}

Elements may be connected to other elements, where the RelatingElement is of equal or higher priority, is generally constructed first, and/ or anchors the RelatedElement.

\subsection{Port connectivity}

Ports on objects may be connected using elements such as cables, ducts, or pipes.

\subsubsection{Interference}

Elements may interfere with other elements, such as cable carriers going through walls. The interference relation enables precedence of interfering elements to be asserted. 


\section{Model View Definition}

\subsection{Overview}

This chapter documents use cases for exchanging information related to electrical disciplines for building design and construction. Industry Foundation Classes (IFC) is the international standard for exchanging Building Information Modeling (BIM) data, which defines hundreds of classes for common use in software, currently supported by approximately 150 applications. A Model View Definition (MVD) defines a subset of the IFC schema that is needed to satisfy one or many Exchange Requirements of the AEC industry. Together with the IFC schema subset, a set of implementation instructions and validation rules, called MVD Concepts, are published. The electronic format to publish the concepts and associated rules is mvdXML. While IFC defines how building information can be represented electronically in general, an MVD defines which information is required for particular scenarios.

\subsection{Exchanges}

Information required at various stages of a building project is organized into Exchanges. Each exchange defines what information is required, optional, inapplicable, or restricted. Application software may support filtering data to be imported or exported for a particular exchange, and contracts for projects may refer to such exchanges to identify the scope and format of information required for delivery.

\subsubsection{Facility occupancy model}

\subsubsection{Requirements}

The facility occupancy model describes the site location, owner's project requirements, and building requirements.

The site location indicates the geographic location for determining climate information, and the legal address for determining the jurisdiction and applicable building codes. 
The owner's project requirements consist of a facility type and a set of space types, each indicating occupancy loads, hours of occupancy, design priorities, and climate control requirements.

\subsubsection{Usage}

The IfcProject indicates overall context including default units. The IfcProject is aggregated by an IfcSite which indicates the geographic location and postal address. The IfcSite is aggregated by an IfcBuilding which indicates overall building requirements in the form of property sets. The IfcProject declares IfcOccupant instances (via IfcRelDeclares) for each class of building occupant which may correspond to a number of people as indicated within the Pset_ActorCommon property set. Each IfcOccupant may have IfcWorkCalendar assignments using IfcRelAssignsToActor. The IfcProject declares IfcWorkCalendar instances (via IfcRelDeclares) for each calendar of occupancy. Each IfcWorkCalendar may have IfcBuilding assignments using IfcRelAssignsToControl.

\subsubsection{Electrical project requirements}

\subsubsection{Requirements}

Electrical project requirements are based on electrical usage criteria as well as equipment determined from usage criteria of other systems such as HVAC and other mechanical systems.

- Occupation: Number of occupants, hours of occupancy, occupancy type

- Cost: Level of finishes

- Architectural: Size of building, number of floors, floor height

- Environment: Heating, cooling, central/unitary

- Illumination: Lighting level, light sources, daylighting, site lighting

- HVAC: Pumps, chillers, fans (power/area for each space)

- Transport: Elevators, escalators

- Control: Automation systems

\subsubsection{Usage}

The IfcBuilding contains various property sets for indicating overall building requirements. 


\subsubsection{Equipment room requirements}

\subsubsection{Requirements}

This exchange captures equipment with significant electrical loads.

\subsubsection{Usage}

Electrical equipment rooms are indicated using IfcSpace with classification source set to 'Omniclass' Table 13 ('Spaces By Function') using identification of '13-8131 11' having the description 'Power Distribution Spaces', which may be further classified according to more specific space usage. As the building spatial hierarchy is not yet defined at this stage, the IfcSpace is declared on the IfcProject using IfcRelDeclares, and has no placement or representation indicated. The IfcSpace may contain equipment (such as IfcChiller, IfcUnitaryEquipment, or IfcElectricDistributionBoard) using IfcRelContainedInSpatialStructure, where the particular locations of the equipment are not yet defined. However, representations of the equipment are required to determine necessary area and volume using the 'Body', 'Footprint', and 'Clearance' representations.

\subsubsection{Space program}

\subsubsection{Requirements}

Spaces are programmed according to size and proximity requirements. Equipment sizes and clearances must be provided.

\subsubsection{Usage}

Spaces are indicated using IfcSpace with classification source set to 'Omniclass' Table 13 ('Spaces by Function'), which may be further classified according to specific space usage. As the building spatial hierarchy is not yet defined at this stage, the IfcSpace is declared on the IfcProject using IfcRelDeclares, and has no placement or representation indicated. Each IfcSpace may contain equipment using IfcRelContainedInSpatialStructure, where the particular locations of the equipment are not yet defined. However, representations of the equipment are required to determine necessary area and volume using the 'Body', 'Footprint', and 'Clearance' representations. 


\subsubsection{Coordinate concept design}

\subsubsection{Requirements}

Once space requirements have been determined, space locations and dimensions are allocated, where they are then adjusted according to specific disciplines to fulfill more detailed requirements.

\subsubsection{Usage}

Spaces are indicated using IfcSpace with classification source set to 'Omniclass' Table 13 ('Spaces by Function'), which may be further classified according to specific space usage. The building spatial hierarchy is defined at this stage, where spaces formerly declared on the IfcProject using IfcRelDeclares are then allocated within the IfcBuilding and IfcBuildingStorey spatial elements using IfcRelAggregates. Placement and representation of each space is indicated, including 'Body' and 'Footprint'. Each IfcSpace may contain equipment using

IfcRelContainedInSpatialStructure, where the particular locations of the equipment are not yet defined. However, representations of the equipment are required to determine necessary area and volume using the 'Body', 'Footprint', and 'Clearance' representations.

\subsubsection{Electrical system types}

\subsubsection{Requirements}

Information is required for selecting main electrical system types.

For devices consuming electricity, the following items are needed:

- Load

- Voltage

- System Type

For devices generating electricity (if any), the following items are needed:

- Capacity

- Output Voltage

- System Type

For devices storing electricity (if any), the following items are needed: 
- Connected Load

- Uptime

For spatial electrical demand, the following items are needed:

- Lighting power density

- Appliance power density

- Equipment power density

\subsubsection{Usage}

Electrical systems are described using IfcDistributionSystem having PredefinedType set to ELECTRICAL. Each top-level system is declared on the IfcProject using IfcRelDeclares. Devices within each system (e.g. IfcChiller, IfcTransportElement, IfcElectricalGenerator) are assigned using the IfcRelAssignsToGroup relationship, where property sets indicate power requirements on devices.

Systems provided by utilities are assigned to the utility company using IfcRelAssignsToActor where an IfcActor identifies the IfcOrganization of the utility having an IfcActorRole set to the user-defined value of 'UTILITY'. Utility-level systems typically contain IfcTransformer and IfcFlowMeter elements.

\subsubsection{Electrical basis of design}

\subsubsection{Requirements}

- Document process model, constraints, formulas, and tables used for making decisions on electrical design.

- Lighting calculations showing required and designed foot-candles

- Estimated panel board loading (including 25\% extra as a projection of future building loads)

- A projection/summation of the panel board loads to justify the sizing of the building transformers

- An economic analysis to justify the selection of either $120 \mathrm{~V} / 208 \mathrm{~V}$ or $277 \mathrm{Y} / 480 \mathrm{~V}$ on the secondary side of the building transformers

- An analysis, for the 277Y/480 V choice, as to whether the step down transformer(s) shall be large central units or smaller units placed throughout the building 
- A short-circuit analysis to determine the AIC rating of the system components.

- A coordination study to determine the circuit breaker settings and system coordination.

\subsubsection{Usage}

To indicate multiple scenarios within a project, each scenario is indicated using IfcWorkPlan declared on the IfcProject using the IfcRelDeclares relationship. Once a plan is approved for usage, it may be nested within an approved IfcProjectOrder. Such work plan may have a nested IfcPerformanceHistory record indicating projected energy usage, which may be nested into sub-components corresponding to subsystems. The particular systems are indicated using IfcDistributionSystem and are assigned to the IfcPerformanceHistory energy projection using the IfcRelAssignsToControl relationship.

\subsubsection{Electrical source of supply}

\subsubsection{Requirements}

Rate structures must be defined for each system type, indicating time intervals and usage.

A process model may be defined coordinating electrical utility service, along with approval requirements.

\subsubsection{Usage}

Each available service is indicated using IfcTaskType indicating a process model with PredefinedType set to OPERATION. Such process model may have nested recurring tasks (IfcTask) via IfcRelNests with time periods indicating when the service applies using IfcTaskTimeRecurring. Costs of each rate structure are indicated by IfcSubContractResourceType where BaseCosts contains one or more IfcCostValue instances. Each IfcSubContractResourceType is assigned to the IfcTaskType or nested IfcTask using the IfcRelAssignsToProcess relationship. The utility (represented by IfcActor) is assigned to the subcontract resource type using the IfcRelAssignsToResource relationship. 


\subsubsection{Electrical space requirements}

\subsubsection{Requirements}

Electrical requirements for each space are elaborated, and each space lists major equipment for consuming, generating, transforming, and storing electricity.

\subsubsection{Usage}

Spaces are indicated using IfcSpace with classification source set to 'Omniclass' Table 13 ('Spaces by Function'), which may be further classified according to specific space usage. The building spatial hierarchy is indicated within the IfcBuilding and IfcBuildingStorey spatial elements using IfcRelAggregates. Placement and representation of each space is indicated, including 'Body' and 'Footprint'. Electrical space requirements are defined on each IfcSpace using property sets, particularly SPARKie_SpaceElectricalRequirements. Each IfcSpace may contain equipment using IfcRelContainedInSpatialStructure, where the particular locations of the equipment are established at this stage. Representations of the equipment are also required to determine necessary area and volume using the 'Body', 'Footprint', and 'Clearance' representations.

\subsubsection{Energy performance}

\subsubsection{Requirements}

Energy usage is estimated based on major equipment, power densities indicated at spaces, and occupancy schedules.

\subsubsection{Usage}

The IfcProject declares one or more IfcPerformanceHistory instances, where the lifecycle phase should be set to DESIGNDEVELOPMENT to indicate development-level estimation precision. Top-level IfcPerformanceHistory instances (typically one) refer to electrical usage at a main utility port, typically corresponding to that on the SINK side of an IfcFlowMeter electrical meter, where such IfcDistributionPort may be assigned to the IfcPerformanceHistory via the IfcRelAssignsToControl relationship. The IfcPerformanceHistory makes use of the Pset_DistributionPortPHistoryCable property set for indicating electrical 
loads at periods of time, where each IfcPropertyReferenceValue points to IfcIrregularTimeSeries.

\subsubsection{Document concept design}

\subsubsection{Requirements}

A completed concept design contains requirements for all disciplines as follows.

- Architectural: For each space, area and relation to other spaces is indicated along with any exterior space requirements.

- Mechanical: For each distribution element, ventilation, thermal loads, and fuel types are indicated.

- Structural: For each element, static weight is indicated as well as dynamic loads (such as from elevator accelerating).

- Construction: For large equipment, installation methods, sequencing, and paths are indicated.

- Costs: Construction and operation costs are established for each system.

\subsubsection{Usage}

The IfcProject contains a full spatial hierarchy, with instances of IfcElement subtypes are contained in spatial elements using IfcRelContainedInSpatialStructure. Property sets indicate qualitative requirements using IfcPropertySet. Structural weight requirements are described by quantity sets holding a 'NetWeight' property. Construction installation paths are indicated via an assigned IfcAnnotation of ObjectType 'Annotation curve' containing an 'Axis' representation, and assigned IfcTask of type MOVE. Costs are indicated by instances of IfcConstructionResource subtypes assigned to IfcTask instances which qualify the type of task for which costs apply.

\subsubsection{Locate electrical loads}

\subsubsection{Requirements}

Light fixtures, outlets, and other devices consuming electricity are placed within the building. The quantity and layout of devices is determined by space classification and electrical power density requirements. 


\subsubsection{Usage}

Electrical devices such as distribution boards, junction boxes, and equipment are positioned within spaces using

IfcRelContainedInSpatialStructure, and embedded or attached to wall coverings or ceiling coverings using IfcRelConnectsElements. Note that IfcWall and IfcSlab indicate structural elements; for example, the blocks of a CMU wall or the framing of a stud wall. Drywall and brick are considering coverings for which IfoCovering is connected using IfcRelCoversBldgElements. Devices embedded within coverings are positioned such that the origin is on the plane of the positive surface, the Axis is perpendicular to the surface, and the RefDirection points in the $+\mathrm{X}$ direction of the surface when installed with normal orientation. Outlets and switches may be aggregated within junction boxes using IfcRelAggregates and hold IfcDistributionPort connections via IfcRelNests linking to the enclosing junction box, which in turn has ports connecting to upstream and downstream devices within the same IfcDistributionCircuit. Loads on devices are indicated using property sets, specifically

Pset_ElectricalDeviceCommon.

\subsubsection{Electrical equipment requirements}

\subsubsection{Requirements}

For each space, the connected load and demand load is determined along with diversity coefficients. Lighting zones may span within a single space or across multiple spaces, for which any geometrically contained light fixtures are considered part of the zone. Circuits are allocated and loads are determined at distribution points. For each device occurrence, equipment is specified in one of three ways: (a) a specific product model; (b) an arbitrary specification with required properties indicated; or (c) an arbitrary specification with multiple acceptable product models indicated. Locations where raceways may later be positioned are allocated using IfcProxy with custom type PROVISIONFORVOID.

One-line diagrams may be derived from this information.

\subsubsection{Usage}

Loads are indicated at IfcDistributionCircuit and IfcDistributionSystem using property sets. Lighting zones are indicated using IfcSpatialZone having PredefinedType of LIGHTING. Device occurrences are indicated by 
instances of IfcDistributionElement subtypes and assigned to IfcDistributionCircuit circuits using IfcRelAssignsToGroup. Type definitions are indicated by instances of IfcDistributionElementType subtypes defined on occurrences using IfcRelDefinesByType. For a type definition that refers to a specific product model, the property set

Pset_ManufacturerTypeInformation is included. For a arbitrary type definition where multiple product models meet the specification, such product model types (IfcDistributionElementType subtype) may be assigned to the specification type (IfcDistributionElementType subtype) using IfcRelAssignsToProduct.

\subsubsection{Electrical equipment rooms}

\subsubsection{Requirements}

For electrical equipment rooms, locations and connectivity of distribution boards and cable carriers are determined.

\subsubsection{Usage}

Distribution boards are indicated using IfcDistributionBoard and are typically placed on walls using IfcRelConnectsElements. Distribution boards that are to be recessed within walls should fill an opening (IfcOpening with type RECESS) using IfcRelFillsElements.

\subsubsection{Lighting layout}

\subsubsection{Requirements}

Lighting may be arranged to indicate specific placement of fixtures. Such fixtures may be attached or hung to surfaces, embedded within coverings, or suspended from ceiling grids. The quantity of fixtures may be determined according to the required lighting power density for the space and the power of each fixture.

\subsubsection{Usage}

Light fixtures are indicated using IfcLightFixture and are contained within spaces using IfcRelContainedInSpatialStructure. Light fixtures that are attached to surfaces should use IfcRelConnectsElements where RelatedElements contains the IfcLightFixture. Light fixtures that are to be recessed within walls should fill an opening (IfcOpening with type 
RECESS) using IfcRelFillsElements. Light fixtures that are placed within ceiling grids should be placed within an IfcGrid that is connected to the IfcCovering corresponding to the ceiling grid.

\subsubsection{Size electrical system}

\subsubsection{Requirements}

Sizing electrical systems involves review and verification of hourly ratings and code requirements for separations between electrical equipment rooms and adjoining spaces (could potentially impact room areas). Information in this exchange is used to size distribution boards, cables, and transformers.

\subsubsection{Usage}

Each IfcCableSegment must be sized to accommodate the rated load, where the NEC defines required wire gauges according to amps. Each IfcDistributionBoard must be sized to accommodate the number of circuits and spare capacity for future expansion. Each IfcTransformer must be sized to accommodate the capacity and voltage of the transformed circuit. Property sets (particularly Pset_ElectricalDevice) indicate electrical characteristics of devices.

\subsubsection{Raceway layout}

\subsubsection{Requirements}

Layout of raceways indicates paths and connectivity of each raceway along with allocation of cables within raceways.

\subsubsection{Usage}

Raceways are indicated using IfoCableCarrierSegment for straight segments and IfcCableCarrierFitting for transitions. A single IfcCableCarrierSegment may be defined with an arbitrary 'Axis' representation indicating the path, where specific segments and fittings are elaborated as assigned objects at finer level of detail using IfcRelAssignsToProduct. Cable carrier segments and fittings are connected together using IfcDistributionPort having PredefinedType of CABLECARRIER. Cables are indicated using IfcCableSegment and are 
contained within an overall IfcCableCarrierSegment using IfcRelAggregates.

\subsubsection{Electrical schematic design}

\subsubsection{Requirements}

Electrical schematic design includes information needed to calculate electrical performance in its entirety. This information may be used to derive schedules for systems, panelboards, equipment, light fixtures, and feeders.

\subsubsection{Usage}

Electrical systems are indicated using IfcDistributionSystem, with individual circuits defined by IfcDistributionCircuit. Panelboard schedules are derived from IfcDistributionBoard and aggregated IfcProtectiveDevice elements for each circuit breaker. Equipment schedules are derived from instances of IfcFlowTerminal subtypes powered by electrical power. Lighting schedules are derived from IfcLightFixture and aggregated IfcLamp elements for each lamp. Feeder schedules are derived from

IfcCableSegment. Property sets indicate specific values at each object.

\subsubsection{Coordinate with other building systems}

\subsubsection{Requirements}

For coordination with other building systems, plans are created showing equipment locations as well as cable routing and connectivity. Electrical Schedules for equipment, fixtures, feeders, panelboards are derived.

\subsubsection{Usage}

Equipment is indicated primarily by subtypes of IfcFlowTerminal, IfcFlowController, and IfcEnergyConversionDevice. Equipment specific to a space is placed within an IfcSpace, while equipment that serves multiple spaces is placed within an IfcBuildingStorey. Cables connecting equipment are attached to ports (IfcDistributionPort) on each device using IfcRelConnectsPorts. 


\subsubsection{Estimate energy usage}

\subsubsection{Requirements}

Energy usage is estimated based on load profiles of major equipment. Estimating energy usage involves calculating connected loads and demand loads at each circuit, and for the overall electrical system. The end result of this calculation may be captured in a Load Letter (format provided by utility), with values specified per project.

- Utilities may require the following information for establishing service:

- Load profile at each device consuming electricity

- Generation profile at each device generating electricity

- Service Location

- Total Area

- Conditioned Space Area

- Type of Heat

- Similar Business: Name, Address, Utility Account Number

- Type of Service: Underground, Overhead, Service Change, Relocation, New, Temporary

- Service Characteristics: Size of Load Wires, Sets of Load Wires Per Phase, Load Wire Type (AL/CU), Terminations: Meterbase/ C.T. Cabinet/ Connection Box/ Switchgear/ Other

- Service Size (amp): 100/150/200/300/400/600/ other

- Voltage: 1P3W-120/240, 3P4W-120/240 (<=200 amps), 3P4W-Wye120/208, 3P4W-Wye-277/480, Other

- Electric Load Excluding Motor Load (kW): Interior Lighting, Exterior Lighting, Electric Cooking, Water Heating, Dryer, Heat Pump, Heat Pump Strip Heat, Computers, Receptacles, Refrigeration, Electric Heat, AC (tons): Data Processing Load Only, Not Including Data Processing

- Electric Motor Load (Except Heating and AC): Phase, Number of Motors, HP, Voltage, Hours of Operation per week

- Estimated Business Operating Time: Hours Per Week, Month Per Year

- Meter Location Desired

- Service Equipment Location Desired

\subsubsection{Usage}

The IfcProject declares one or more IfcPerformanceHistory instances, where the lifecycle phase should be set to DESIGNSCHEMATIC to indicate schematic-level estimation precision. Top-level 
IfcPerformanceHistory instances (typically one) refer to electrical usage at a main utility port, typically corresponding to that on the SINK side of an IfcFlowMeter electrical meter, where such IfcDistributionPort may be assigned to the IfcPerformanceHistory via the IfcRelAssignsToControl relationship. The IfcPerformanceHistory makes use of the Pset_DistributionPortPHistoryCable property set for indicating electrical loads at periods of time, where each IfcPropertyReferenceValue points to IfcIrregularTimeSeries.

\subsubsection{Facility spatial configuration}

\subsubsection{Requirements}

This exchange enables an architect to revise the facility spatial configuration plans based on the results of the coordination that took place at the end of Design Schematic. Required information includes:

- Spatial Elements (Buildings, Levels, Spaces, etc.)

- Building Elements (Walls, Slabs, Doors, Windows, etc.)

- Distribution Elements (Electrical, HVAC, Plumbing, etc.)

- Spatial Zones

- Systems \& Circuits

- Connectivity (Space Boundaries, Ports, Connections, Interferences)

- Actors \&Assignments

\subsubsection{Usage}

Project participants responsible for particular systems are indicated using IfcActor with assignments through IfcRelAssignsToActor.

Interferences with other building elements are indicated using IfcRelInterferesElements, where priorities may be indicated at such intersection.

\subsubsection{Electrical supply requirements}

\subsubsection{Requirements}

In this exchange, an electrical engineer uses the product type templates, updated plans, and other discipline information to determine total electrical supply requirements. For each electrical device, compatible product types are selected for each product occurrence (or if required, three compatible product types are selected that are suitable). The project delivery 
method may require the owner's approval for final product selection. The total electrical supply requirements are calculated on each circuit according to connected load, demand load, and diversity factor.

\subsubsection{Usage}

For each electrical device, the specified type or range of types is defined using IfcRelDefinesByType. Overall electrical supply requirements are established at property set on IfcDistributionSystem of type ELECTRICAL.

\subsubsection{System loads}

\subsubsection{Requirements}

Loads are calculated by rolling up elements, circuits, and systems as follows:

- Element: Load, Time-phased load

- Circuit: Connected Load, Demand Load, Time-phased load

- System: Connected Load, Demand Load, Time-phased load, Diversity Coefficient

\subsubsection{Usage}

Devices are indicated using instances of IfcDistributionElement subtypes. Circuits are indicated using IfcDistributionCircuit having PredefinedType set to ELECTRICAL and have devices assigned using IfcRelAssignsToGroup. Systems are indicated using IfcDistributionSystem having PredefinedType set to ELECTRICAL and have circuits nested using IfcRelNests.

\subsubsection{Cabling schematic}

\subsubsection{Requirements}

This exchange provides detailed information for connectivity and placement of devices cables, including the following:

- Switch: Location, Lighting Load, Controls

- Outlet: Location, Appliance Load

- Cable Segment: Location, Connections, Load, Length, Wiring method (EMT/ ENT/ MC/ Rigid/ etc.) 
- Switchgear/Panels

- J unction Box: Location (note: may not be at this level of detail)

- Life Safety devices / control panels: Location

- Lighting: Location

- Telecom: Location

- Generating Equipment: controls, transfer switches, interconnects: Location

All products may have defined types indicating Manufacturer, Model, and Specifications. Such types may also have assigned tasks and resources for procurement, where resource types indicate Supplier, Location, and Cost.

\subsubsection{Usage}

All electrical devices are connected together via ports (IfcDistributionPort having PredefinedType of CABLE and SystemType of ELECTRICAL), where the relationship IfcRelConnectsPorts has RelatingPort set to the power source (having FlowDirection of SOURCE) and RelatedPort set to the load (having FlowDirection of SINK). Product types are indicated via subtypes of IfcDistributionElementType. Costs rates for product types are indicated via subtypes of IfcConstructionResourceType assigned to IfcTaskType assigned to the IfcDistributionElementType. The task type qualifies the scenario for which the cost applies.

\subsubsection{Redistribute circuits}

\subsubsection{Requirements}

This exchange is used to rebalance circuits based on calculations derived from the cabling schematic. Circuits with loads above a maximum factor may be split into separate circuits. Circuits with loads below a minimum factor may be combined.

\subsubsection{Usage}

Each circuit is represented using IfcDistributionCircuit with PredefinedType set to ELECTRICAL. Loads may be calculated according to information at property sets, particularly

Pset_ElectricalDeviceCommon. 


\subsubsection{Cabling and equipment size}

\subsubsection{Requirements}

Based on final allocation of circuits, cabling and equipment sizes may be adjusted.

\subsubsection{Usage}

Cable segments are indicated using IfcCableSegment, where cable size information is indicated via IfcMaterialProfileSet.

Raceways are indicated using IfcCableCarrierSegment, where cross section is indicated via IfcMaterialProfileSet.

\subsubsection{Architectural light fixtures}

\subsubsection{Requirements}

For this exchange, the architect selects specific light fixture models (or an approved list from several manufacturers).

\subsubsection{Usage}

Light fixture occurrences are indicated by IfcLightFixture, where the selected model is defined by IfcLightFixtureType defined using IfcRelDefinesByType. To indicate multiple accepted models, the top-level model (IfcLightFixtureType) indicates an abstract template (not having a model defined via Pset_ManufacturerTypeInformation) and has candidate types assigned using IfcRelAssignsToProduct. Each candidate type has model information defined via the Pset_ManufacturerTypeInformation property set.

\subsubsection{Product type specifications}

\subsubsection{Requirements}

For this exchange, the engineer selects specific electrical equipment models (or an approved list from several manufacturers).

\subsubsection{Usage}

Electrical equipment occurrences are indicated by various IfcDistributionElement subtypes, where the selected model is defined by 
IfcDistributionElementType defined using IfcRelDefinesByType. To indicate multiple accepted models, the top-level model (IfcDistributionElementType) indicates an abstract template (not having a model defined via Pset_ManufacturerTypeInformation) and has candidate types assigned using IfcRelAssignsToProduct. Each candidate type has model information defined via the Pset_ManufacturerTypeInformation property set.

\subsubsection{Document coordinated design}

\subsubsection{Requirements}

The coordinated design contains full detail for all electrical devices and their placement and interaction with other services within the building.

\subsubsection{Usage}

Electrical elements are defined using subtypes of IfcDistributionElement, with ObjectPlacement and Representation set for all instances. Electrical distribution ports are indicated using IfcDistributionPort, where all ports having FlowDirection of SINK must be connected. (Source ports may not necessarily be connected such as open outlets or the last junction box within a circuit.) 


\section{Conclusions}

In developing Model View Definitions, the challenge is to extract detailed information from industry experts yet find commonalities that could be applied generally across varying project delivery methods, participants, and localities. During this project there were varying levels of input. Some experts would work within the assumptions of the preliminary structure, others would alter various steps, and some created new process diagrams from scratch. Each party had different project delivery methods. Therefore, dependencies were factored out by making each exchange role-based, not contract-based. Achieving this level of granularity required many more exchanges than traditionally used in IFC Model View Definitions. For example, information sent to a utility for obtaining rate structures and connection information is one specific exchange, rather than being lumped into a higher level category such as "early design." The definition of rolebased exchanges supports a variety of project delivery methods. Where possible, exchanges were aggregated into higher levels when appropriate.

Once each exchange was defined, the specific information needed down to the attribute-level of detail was described, leveraging the existing scope of the IFC data model where possible. While most product geometry information was already well-defined within IFC version $2 \times 3$ and implemented by many vendors, there were many concepts that required some of the lesser-supported IFC data structures and some that required the expanded MEP scope in IFC version 4 to achieve adequate levels of detail. There were also many cases of data constructs already in possible in the IFC schema but never detailed in the documentation - some examples of these include indicating multiple approved product types, ceiling grids for lighting layout, and indicating projected power usage at different times of day throughout a year. While realizing that many of these concepts were not supported by existing COTS software, the MVD has been defined to allow partial compliance for now, but with allowances to later relax or replace some requirements after testing models produced by existing software.

In detailing functional parts used within the model view, this project also contributed new concepts back to IFC4 that appeared to have wider uses in other disciplines (as IFC4 was not yet finalized at the time). For example, a functional part for generically mapping data to spreadsheets was 
formalized to support common tables such as lighting schedules, while also supporting other Model View Definitions such as COBie; this functional part also involved advancing the parametric capability of IFC with the ability to generically reference object attributes. Similarly, as details on connections between equipment were elaborated, such uses also made their way into expanded port specifications within IFC4.

Once the Model View Definition was complete, IFC files were tested with the mvdXML electronic validation format and noted concepts that were supported by existing software and those requiring new functionality. There were some very basic limitations such as not capturing the physical building address, which is required for determining applicable codes and utilities, and more complex limitations such as detailing projected utility usage. In trying to find a balance that would encourage faster adoption by vendors, critical concepts were strongly enforced while others were relaxed by making certain attributes optional.

Going forward, the IFC4 release and supporting technology has provided for integrated Model View Definitions where the IFC specification and all published Model View Definitions will be made available online in an integrated form. This will enable developers of IFC to instantly cross-reference usage of entities across multiple model views and to define templates only once where they are re-used across model views. The supporting mvdXML technology provides for computer-interpretable validation, content filtering, sub-schema generation, and data adaptation. This enables new IFC software vendors to support information models with a substantially lower barrier of entry, and enables established software vendors with full IFC support to handle new Model View Definitions automatically without additional work. This Model View Definition is one of the first to leverage the growing ecosystem of mvdXML and has influenced the future direction of IFC with the various supporting concepts. 


\section{References}

East, E.W. 2007. Construction Operations Building Information Exchange (COBIE), Requirements Definition and Pilot Implementation Standard, ERDC/ CERL TR07-30, US Army Corps of Engineers, http://www.wbdg.org/pdfs/erdc_cerl_tr0730.pdf August 2007.

East, E.W. 2012a. "Construction Operations Building Information Exchange (COBie)," http://www.wbdg.org/resources/cobie.php 6 August 2012.

East, E.W. 2012b. "Electrical System information exchange (Sparkie)," http://www.buildingsmartalliance.org/index.php/projects/activeprojects/178 17 August 2012.

East, E.W. 2012c. "Equipment Layout information exchange (ELie)," http://www.buildingsmartalliance.org/index.php/projects/activeprojects/114 17 August 2012.

East, E.W., Danielle Love and Nicholas Nisbet. 2010. "A Life-Cycle Model for Contracted Information Exchange." Proceedings of the CIB W78 2010: 27th International Conference-Cairo, Egypt, 16- 18 November 2010.

East, E.W. "COBie Guide web page," National Institute of Building Sciences, accessed 19 J une 2013, http://www.nibs.org/?page=bsa_cobieguide.

Fallon, Kristine K. and Mark E. Palmer. 2007. General Buildings Information Handover Guide: Principles, Methodology and Case Studies. NISTIR 7417, National Institute of Standards and Technology, August 2007.

Hietanen, J . 2008. IFC Model View Definition Format," http://www.iaitech.org/downloads/accompanyingdocuments/formats/MVD_Format_V2_Proposal_080128.pdf.

Wix, J ., ed. 2007. “Information Delivery Manual: Guide to Components and Development Methods,"

http://www.iai.no/idm/idm_resources/idm_methods_guides/IDM2_Methodology_20071022.p df. 


\section{REPORT DOCUMENTATION PAGE}

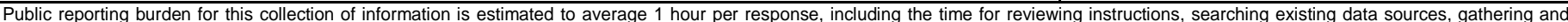

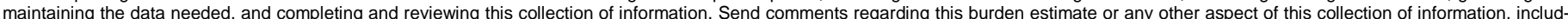

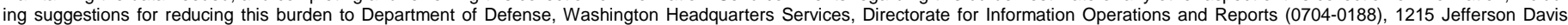

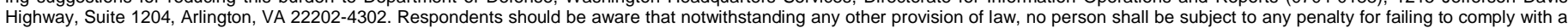
collection of information if it does not display a currently valid OMB control number. PLEASE DO NOT RETURN YOUR FORM TO THE ABOVE ADDRESS.

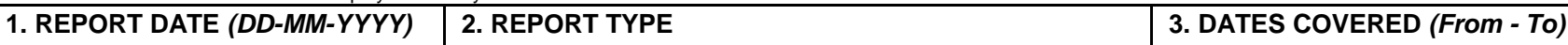

June 2013 Final

4. TITLE AND SUBTITLE

Ontology for Life-Cycle Modeling of Electrical Distribution Systems: Model View Definition

5a. CONTRACT NUMBER

CRADA-07-CERL-02

5b. GRANT NUMBER

5c. PROGRAM ELEMENT NUMBER

622784 T41

\section{AUTHOR(S)}

Tim Chipman, Kristine K. Fallon, Robert A. Feldman, Gregory Williams, and

Omobolawa Fadojutimi

5d. PROJECT NUMBER

5e. TASK NUMBER

5f. WORK UNIT NUMBER

7. PERFORMING ORGANIZATION NAME(S) AND ADDRESS(ES)

Kristine Fallon Associates, Inc.

8. PERFORMING ORGANIZATION REPORT NUMBER

11 E. Adams Street, Suite 1100

Chicago, IL 60603

9. SPONSORING / MONITORING AGENCY NAME(S) AND ADDRESS(ES)

US Army Engineer Research and Development Center

Construction Engineering Research Laboratory

P.O. Box 9005

Champaign, IL 61826-9005

10. SPONSOR/MONITOR'S ACRONYM(S)

ERDC-CERL

11. SPONSOR/MONITOR'S REPORT NUMBER(S)

ERDC/CERL CR-13-2

\section{DISTRIBUTION / AVAILABILITY STATEMENT}

Approved for public release; distribution is unlimited.

\section{SUPPLEMENTARY NOTES}

\section{ABSTRACT}

Previous efforts by the US Army Engineer Research and Development Center, Construction Engineering Research Laboratory (ERDC-CERL) to develop a life-cycle building model have resulted in the definition of a "core" building information model that contains general information describing facility assets such as spaces and equipment. To describe how facility assets (i.e., components) function together, information about assemblies of assets and their connections must also be defined. The definitions of assets, assemblies, and connections for the various building-information domains are discipline-specific.

The work documented here addresses the process flow and data exchange requirements for the design of electrical distribution systems in typical Army facilities. This ontology advances the state of the art by defining an Industry Foundation Class (IFC) Model View for electrical system design supporting end users in developing compliant BIM models suggesting potential areas of automation in electrical system design.

\section{SUBJECT TERMS}

information exchange, electrical systems, product data templates, guide specifications, Construction Operations Building information exchange (COBie), Building Information Modeling (BIM)

\section{SECURITY CLASSIFICATION OF:}

\begin{tabular}{|l|r|}
\hline a. REPORT & b. ABSTRACT \\
Unclassified & Unclassified
\end{tabular}

17. LIMITATION OF ABSTRACT

18. NUMBER
OF PAGES
71

\section{9a. NAME OF RESPONSIBLE} PERSON

19b. TELEPHONE NUMBER (include area code) 